\title{
INFINITE LOOP SPACE THEORY
}

\author{
BY J. P. MAY ${ }^{1}$
}

Introduction. The notion of a generalized cohomology theory plays a central role in algebraic topology. Each such additive theory $E^{*}$ can be represented by a spectrum $E$. Here $E$ consists of based spaces $E_{i}$ for $i \geqslant 0$ such that $E_{i}$ is homeomorphic to the loop space $\Omega E_{i+1}$ of based maps $S^{1} \rightarrow E_{i+1}$, and representability means that $E^{n} X=\left[X, E_{n}\right]$, the Abelian group of homotopy classes of based maps $X \rightarrow E_{n}$, for $n \geqslant 0$. The existence of the $E_{i}$ for $i>0$ implies the presence of considerable internal structure on $E_{0}$, the least of which is a structure of homotopy commutative $H$-space. Infinite loop space theory is concerned with the study of such internal structure on spaces.

This structure is of interest for several reasons. The homology of spaces so structured carries "homology operations" analogous to the Steenrod operations in the cohomology of general spaces. These operations are vital to the analysis of characteristic classes for spherical fibrations and for topological and PL bundles. More deeply, a space so structured determines a spectrum and thus a cohomology theory. In the applications, there is considerable interplay between descriptive analysis of the resulting new spectra and explicit calculations of homology groups.

The discussion so far concerns spaces with one structure. In practice, many of the most interesting applications depend on analysis of the interrelationship between two such structures on a space, one thought of as additive and the other as multiplicative.

The purpose of this talk is to give my view of the present state of infinite loop space theory, with emphasis on the intuitions behind the main concepts. There will be no formal statements, no proofs, very few complete definitions, and a general disregard of technical niceties. The details are now all written down, largely in [20], [45], and [48]. As these references indicate, this is not a historical survey, and I shall have little to say about the alternative theoretical approaches applicable to various portions of the material presented. ${ }^{2}$ For the applications, it is sufficient, and necessary, to have a fully coherent framework, and my primary concern is to explain how the classifying spaces of geometric topology, the spectra of algebraic and topological $K$-theory, and Thom spectra appear and interact within one such framework and to show how the general abstract machinery is used to crank out explicit concrete calculations.

This is an expanded version of an invited address given at the annual meeting of the Society in San Antonio, Texas, on January 25, 1976; received by the editors July 2, 1976.

AMS (MOS) subject classifications (1970). Primary 55-02, 55B20, 55D35, 55F40; Secondary 55B15, 55D40, 55E50, 55F50, 18C15, 18D10, 18F25, $18 \mathrm{H} 10$.

'The author was partially supported by NSF grant GP-29075.

${ }^{2}$ Frank Adams is presently preparing a survey of this general area. 
To give proper credits without interrupting the exposition (and to indicate where in the references cited above the details may be found), I have ended most sections with brief historical notes.

The following diagram depicts some of the main concepts and the functors relating them. The word "coherent" in the previous paragraph refers, in part, to analysis of the various commutativity relations in the diagram. We shall refer to this diagram as the schematic picture, and we shall orient our discussion of the general theory with respect to it.

PERMUTATIVE

CATEGORIES

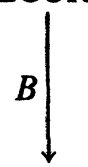

BIPERMUTATIVE

CATEGORIES

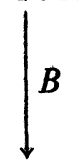

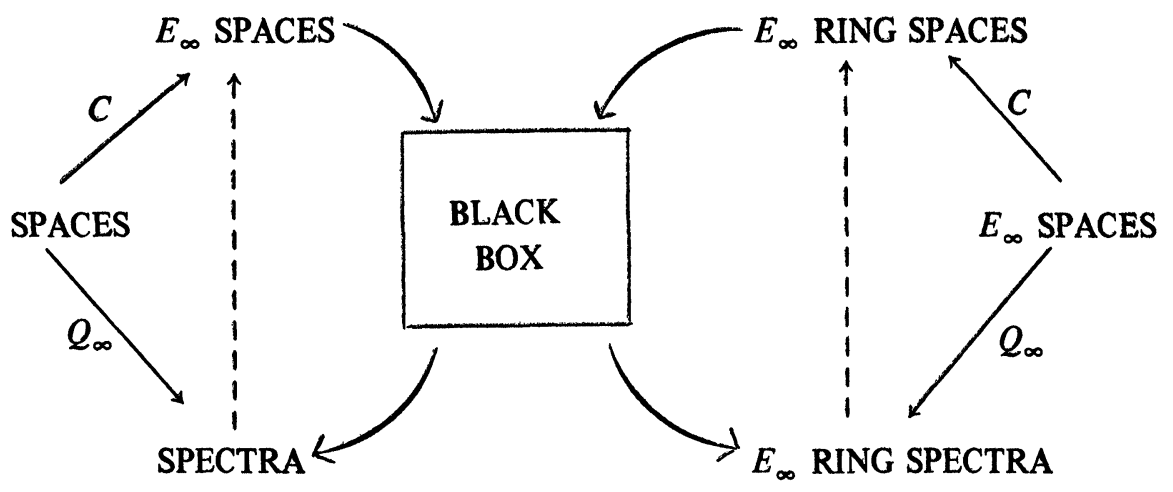

CONTENTS

Chapter I. Additive infinite loop space theory

1. Spectra, infinite loop spaces, and the functor $Q_{\infty}$

2. Operads and their actions; $\mathfrak{R}, \mathfrak{X}$, and $\mathfrak{U}_{n}$

3. Monads and their actions; $M, N$, and $C_{n}$

4. The approximation theorem

5. The recognition principle

6. Homotopy invariance of the recognition principle

7. $\mathscr{I}_{*}$ functors and classifying spaces; $\mathfrak{L}$

8. Symmetric monoidal and permutative categories; $\mathscr{D}$

Chapter II. Multiplicative infinite loop space theory

9. Operad pairs and their actions; $(\Re, \Re)$

10. Symmetric bimonoidal and bipermutative categories; $(\mathscr{D}, \mathscr{D})$

11. $E_{\infty}$ ring spectra; $\left(\mathcal{K}_{\infty}, \mathfrak{E}\right)$

12. The recognition principle

13. $g_{*}$ prefunctors and Thom spectra

14. Orientation theory and $B(G ; E)$

Chapter III. Descriptive analysis of infinite loop spaces

15. General remarks on methodology

16. Transfer and results about $B S O$

17. The uniqueness of deloopings 
18. The $k O$ orientations of Spin and $S$ Top bundles

19. Brauer lift, Frobenius, and finite fields

Chapter IV. Homological analysis of infinite loop spaces

20. Homology operations on $E_{\infty}$ spaces

21. The Dyer-Lashof algebra; $H_{*} C X$ and $H_{*} Q X$

22. The homology of $E_{\infty}$ ring spaces; $H_{*} C\left(X^{+}\right), H_{*} Q\left(X^{+}\right), H_{*} S F$

23. The homology of matrix groups; $B C \rightarrow B S F \rightarrow B J_{\otimes}^{\delta}$

24. The homology of $n$-fold loop spaces

Conclusion

\section{Chapter I. AdDitive infinite loop SPaCE THEORY}

1. Spectra, infinite loop spaces, and the functor $Q_{\infty}$. We have defined a spectrum $E$ to be a sequence of spaces $E_{i}$ with $E_{i}$ homeomorphic to $\Omega E_{i+1}$. Maps of spectra are sequences $f_{i}: D_{i} \rightarrow E_{i}$ such that $f_{i}$ agrees under the given homeomorphisms with $\Omega f_{i+1}$. Spaces of the homotopy type of some $E_{0}$ and maps in the homotopy class of some $f_{0}$ are called infinite loop spaces and infinite loop maps.

The suspension $\Sigma X$ of a based space $X$ is the smash product $X \wedge S^{1}$, where $X \wedge Y$ is the quotient of $X \times Y$ by the wedge, or 1-point union, $X \vee Y$. The $n$th stable homotopy group $\pi_{n}^{s} X$ of $X$ is the direct limit of the groups $\pi_{n+j} \Sigma^{j} X$.

There is a stabilization functor $Q$ from spaces to spaces specified by $Q X=$ ind $\lim \Omega^{j} \Sigma^{j} X ; \pi_{n} Q X=\pi_{n}^{s} X$, and the natural map $X \rightarrow Q X$ induces the stabilization homomorphism upon passage to homotopy groups. Since $\Omega Q \Sigma X \cong Q X$, there is a stabilization functor $Q_{\infty}$ from spaces to spectra specified by

$$
\left(Q_{\infty} X\right)_{i}=Q \Sigma^{i} X=\text { ind } \lim \Omega^{j} \Sigma^{i+j} X .
$$

The sphere spectrum $Q_{\infty} S^{0}$ and its zeroth space $Q S^{0}$ will be central to our discussion. The elements of $Q S^{0}$ may be thought of as maps of an infinite sphere to itself. The groups $\pi_{n}^{s}=\pi_{n} Q S^{0}$ are the stable homotopy groups of spheres, the determination of which is, of course, one of the hardest and most important of the unsolved problems in algebraic topology.

Notes. The notion of spectrum here is not the classical one. See [48, II] for a summary and [49] for the details of a treatment of stable homotopy theory based on this notion. The spaces $Q X$ seem to have been introduced by Dyer and Lashof [22].

2. Operads and their actions; $\mathscr{N}, \mathcal{T}$, and $\bigcup_{n}$. We require a convenient language in which to describe the internal structure on an infinite loop space $E_{0}$ given by the existence of the spaces $E_{i}$. Since $E_{0} \cong \Omega E_{1}$, composition of loops gives a product $E_{0} \times E_{0} \rightarrow E_{0}$; that is, given two loops $S^{1} \rightarrow E_{1}$, we can use one on the first half of $S^{1}$ and the other on the second half to obtain a new loop. Consideration of the associativity of this operation reveals that the use of $\frac{1}{2}$ here is an arbitrary choice. We could just as well have used one loop on the first $\frac{1}{4}$ of $S^{1}$ and the other on the last $\frac{3}{4}$ for example. Moreover, $E_{0} \cong \Omega^{n} E_{n}$, and this gives $n$ different coordinates which can be used to define a product, all of these products being homotopic. We are led to introduce a space of products, a space of 3 -fold products $E_{0} \times E_{0} \times E_{0} \rightarrow E_{0}$, and so on. 
These spaces of operations will encode the internal structure on $E_{0}$ coming from the $E_{i}$.

An appropriate formalism is given by the notion of an operad. An operad e consists of spaces $e(j)$ for $j \geqslant 0$, to be thought of as spaces of $j$-ary operations. $\mathcal{C}(0)$ is a point, $\mathcal{C}(1)$ contains a unit element 1 , there is a right action of the symmetric group $\Sigma_{j}$ on $\mathcal{C}(j)$, and there are maps

$$
\gamma: \mathcal{C}(k) \times \mathfrak{C}\left(j_{1}\right) \times \cdots \times \mathfrak{C}\left(j_{k}\right) \rightarrow \mathfrak{C}\left(j_{1}+\cdots+j_{k}\right) .
$$

These data are subject to a number of axioms, which will be omitted in favor of a conceptual reformulation to be given shortly.

An action $\theta$ of $e$ on a space $X$ consists of $\Sigma_{j}$-equivariant maps $\theta_{j}$ : $\mathcal{Q}(j) \times X^{j} \rightarrow X$ (where $\Sigma_{j}$ acts diagonally on the left and trivially on the right) subject to certain axioms. $\theta_{0}$ is to be interpreted as the inclusion of the basepoint or identity element $*$ in $X$. The unit $1 \in \mathcal{E}(1)$ determines the identity map of $X$, the equivariance of the $\theta_{j}$ keeps track of permutations of variables, and the maps $\gamma$ are so related to composition as to keep track of iterated products.

These notions are not unfamiliar. There is an operad ơ with : : $(j)=\Sigma_{j}$. An $\Re$-space is just a monoid (that is, an associative $H$-space with unit). The elements of $\Sigma_{j}$ simply record the $j$ ! ways of writing words with $j$ distinct letters. Again, there is an operad $\mathfrak{N}$ with each $\Re(j)$ a single point. An $\mathfrak{X}$-space is just a commutative monoid.

An operad $\mathcal{C}$ is said to be an $E_{\infty}$ operad if each $\mathcal{C}(j)$ is a contractible space with a free $\Sigma_{j}$-action. The orbit space $\mathcal{C}(j) / \Sigma_{j}$ is then an Eilenberg-Mac Lane space $K\left(\Sigma_{j}, 1\right)$. An $E_{\infty}$ space is a $\bigodot$-space where $\bigodot$ is any $E_{\infty}$ operad. It would be most inconvenient to restrict attention to any particular example of an $E_{\infty}$ operad: we shall have three very different ones on hand before we are through.

Returning to our motivating example, we must construct an $E_{\infty}$ operad which acts naturally on infinite loop spaces. Let $\varrho_{n}(j)$ be the space of $j$-tuples of little $n$-cubes with disjoint interiors. Here by a "little $n$-cube" we mean a linear embedding $I^{n} \rightarrow I^{n}$ (with parallel axes). With the appropriate data, $\stackrel{E}{n}_{n}$ is an operad. Via little cubes with last coordinate the identity map, $\vec{E}_{n}$ is a suboperad of $e_{n+1}$, and there results a direct limit operad $E_{\infty}$. $E_{n}(j)$ is $\Sigma_{j}$-equivariantly equivalent to the configuration space of $j$-tuples of distinct points of $R^{n}$ and is $(n-2)$-connected; $e_{\infty}$ is an $E_{\infty}$ operad.

$\bigcup_{n}$ acts naturally on $n$-fold loop spaces. The requisite maps $\theta_{n, j}: \varrho_{n}(j) \times$ $\left(\Omega^{n} X\right)^{j} \rightarrow \Omega^{n} X$ can be described pictorially as follows (with $j=3$ ):

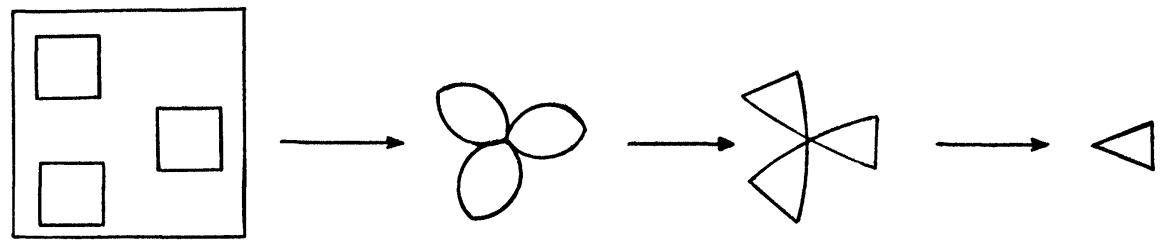

In the first map, we collapse all points of $I^{n}$ not in the image of three given little $n$-cubes to a single basepoint. The second space is then the wedge of three $n$-spheres, and we can use three given maps $S^{n} \rightarrow X$ to map this space to the wedge of three copies of $X$. Finally, we apply the folding map, the identity on each copy of $X$. 
For a spectrum $E$, the actions $\theta_{n}$ of $e_{n}$ on $E_{0} \cong \Omega^{n} E_{n}$ together specify an action of $\mathcal{C}_{\infty}$ on $E_{0}$. Thus an infinite loop space is an $E_{\infty}$ space. This gives the dotted arrow from spectra to $E_{\infty}$ spaces in our schematic picture.

NotEs. See $[45, \S \S 1,3-5]$ for details. The notion of an operad was obtained by deletion of extraneous structure from that of a PROP. The latter notion was introduced by Adams and Mac Lane [37] but was first topologized by Boardman and Vogt [13], [14]; this deceptively simple sounding change of point of view was the essential starting point of this area of topology. The invention of the little cubes operads and the definition of their actions on iterated loop spaces are also due to Boardman and Vogt.

3. Monads and their actions; $M, N$, and $C_{n}$. While the notion of an operad is naturally dictated by the geometry, exploitation of this concept requires a simpler notion for which an action is not a sequence of maps but a single map. The basic idea is the description of algebraic structure by means of maps from free objects to objects. For example, an action of a monoid $G$ on a space $X$ is a map $G \times X \rightarrow X$ such that the appropriate associativity and unity diagrams commute.

A monad $(C, \mu, \eta)$ in a category $\mathscr{T}$ is a functor $C: \mathfrak{T} \rightarrow \mathscr{T}$ together with natural transformations $\mu: C C \rightarrow C$ and $\eta: 1 \rightarrow C$ such that the following diagrams commute for all $X \in \mathcal{T}$ :

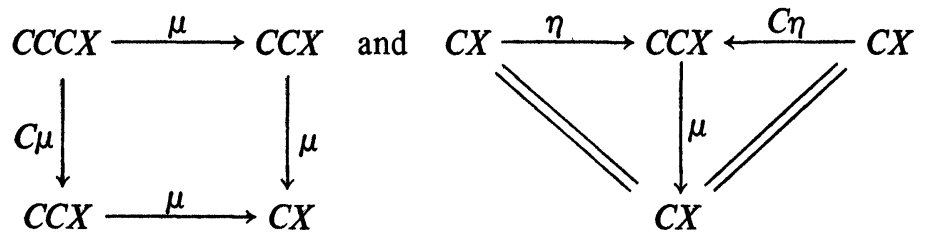

An action of a monad $C$ on an object $X$ is a map $\theta: C X \rightarrow X$ such that the following diagrams commute:

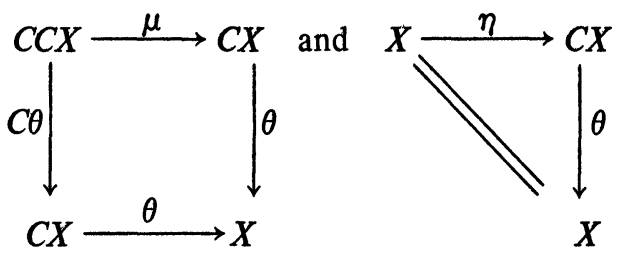

In the present additive theory, the ground category $T$ will just be the category of (suitably nice) based spaces.

We claim that an operad $\mathcal{C}$ determines a monad $C$ in such a way that the notions of $\mathcal{C}$-space and of $C$-space are equivalent. Indeed, for a space $X$, the free $C_{\text {-space }} C X$ is specified by

$$
C X=\amalg_{j>0} e(j) \times_{\Sigma_{j}} X^{j} /(\approx),
$$

where II denotes disjoint union and the equivalence is given by appropriate basepoint identifications (of the form $\left(\sigma_{i} c, y\right) \approx\left(c, s_{i} y\right)$ for certain maps $\sigma_{i}$ : $\mathcal{C}(j) \rightarrow \mathcal{C}(j-1)$, where $s_{i}: X^{j-1} \rightarrow X^{j}$ inserts the basepoint in the $i$ th position). The maps $\mu: C C X \rightarrow C X$ and $\eta: X \rightarrow C X$ are obtained by use of the structural maps $\gamma$ and the unit $1 \in \mathcal{C}(1)$ of the operad. The missing axioms in 
the definition of an operad are precisely the conditions necessary to ensure that $(C, \mu, \eta)$ is in fact a well-defined monad in $\mathcal{T}$. The maps $\theta_{j}$ of an action of $\mathcal{C}$ on $X$ together determine a map $\theta: C X \rightarrow X$, and the missing axioms in the definition of an action by an operad are precisely the conditions neces. sary to ensure that $\theta$ is an action of $C$ on $X$.

The monads $M$ and $N$ associated to $\Re$ and $\Re$ are familiar old constructions in algebraic topology. $M X$ is the James reduced product construction, or free monoid, on $X$ and is equivalent to $\Omega \Sigma X$ if $X$ is connected. $N X$ is the infinite symmetric product, or free commutative monoid, on $X$ and is equivalent to $X_{n \geqslant 1} K\left(H_{n} X, n\right)$ if $X$ is connected.

For any operad $\mathcal{C}, C S^{0}=\amalg \mathcal{C}(j) / \Sigma_{j}$. Thus, if $\bigcup$ is an $E_{\infty}$ operad, $C S^{0}$ is a disjoint union of $K\left(\Sigma_{j}, 1\right)$ 's. We shall later need a structured way of comparing two $E_{\infty}$ operads and their associated monads. This is obtained simply by

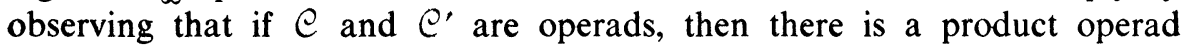
$\mathcal{C} \times \mathcal{C}^{\prime}$ with $j$ th space $\mathcal{C}(j) \times \mathcal{C}^{\prime}(j)$. We write $C \times C^{\prime}$ for the associated monad. When $C$ and $C^{\prime}$ are $E_{\infty}$ operads, the projections from $\left(C \times C^{\prime}\right)(X)$ to $C X$ and to $C^{\prime} X$ are equivalences.

The monads $C_{n} X$ are of particular interest. Note that $\Omega^{n} \Sigma^{n}$ is a monad with $\mu: \Omega^{n} \Sigma^{n} \Omega^{n} \Sigma^{n} X \rightarrow \Omega^{n} \Sigma^{n} X$ being the $n$th loop of the evaluation map and $\eta$ : $X \rightarrow \Omega^{n} \Sigma^{n} X$ being the natural inclusion. Define $\alpha_{n}: C_{n} X \rightarrow \Omega^{n} \Sigma^{n} X$ to be the composite

$$
C_{n} X \stackrel{C_{n} \eta}{\rightarrow} C_{n} \Omega^{n} \Sigma^{n} X \stackrel{\theta_{n}}{\rightarrow} \Omega^{n} \Sigma^{n} X .
$$

It is a pleasant formal fact that $\alpha_{n}: C_{n} \rightarrow \Omega^{n} \Sigma^{n}$ is then a morphism of monads.

Notes. See $[45, \S \S 2,3,5]$ for details. The utility of monads in infinite loop space theory was first recognized by Beck [12].

4. The approximation theorem. There is a far more substantial topological reason for interest in the maps $\alpha_{n} . \Omega^{n} \Sigma^{n} X$ is a huge and unwieldy space, not amenable to direct homotopical analysis. The spaces $C_{n} X$ are much more tractable. Indeed, $C_{n} X$ is filtered by successive cofibrations, with $F_{k} C_{n} X$ being the image of $\amalg_{j=0}^{k} \bigodot_{n}(j) \times X^{j}$. The successive quotients $F_{k} C_{n} X / F_{k-1} C_{n} X$ are the "equivariant half-smash products"

$$
e_{n}^{k} X=\bigodot_{n}(k) \propto_{\Sigma_{k}} X^{[k]} \equiv \bigodot_{n}(k) \times_{\Sigma_{k}} X^{[k]} / \bigodot_{n}(k) \times_{\Sigma_{k}} *
$$

where $X^{[k]}$ is the $k$-fold smash product of $X$ with itself. The approximation theorem asserts that the map $\alpha_{n}: C_{n} X \rightarrow \Omega^{n} \Sigma^{n} X$ is an equivalence if $X$ is connected and that $\alpha_{\mathrm{n}}$ is a "group completion" in general.

Here by a group completion of an $H$-space $Y$ we understand an $H$-map g: $Y \rightarrow Z$ such that $\pi_{0} Z$ is a group and $g_{*}: H_{*}(Y ; k) \rightarrow H_{*}(Z ; k)$ is a localization of the Pontryagin ring $H_{*}(Y ; k)$ at its multiplicative submonoid $\pi_{0} X$ for any commutative coefficient ring $k$. Intuitively then, $Z$ is obtained from $Y$ by adjoining inverses to components. If $\pi_{0} Y$ is itself a group, then $g$ is necessarily an equivalence.

Homotopical exploitation of the approximation theorem has barely begun. We digress to cite two applications. First, we have a generalized EHP. sequence. Let $X$ be $(q-1)$-connected, $q \geqslant 2$. Then $e_{n}^{k} X$ is $(k q-1)$ connected and the inclusion of $F_{2} C_{n} X$ in $C_{n} X$ induces an isomorphism on homotopy groups in dimensions $i<3 q-1$. Since $\left(F_{2} C_{n} X, X\right)$ is $(2 q-1)$ - 
connected, the projection $\left(F_{2} C_{n} X, X\right) \rightarrow\left(e_{n}^{2} X, *\right)$ induces an isomorphism on homotopy groups in dimensions $i<3 q-2$ (this being a standard consequence of the homotopy excision theorem). Thus, in this range, the homotopy exact sequence of the pair $\left(F_{2} C_{n} X, X\right)$ yields an exact sequence of the form

$$
\ldots \rightarrow \pi_{i} X \stackrel{E}{\rightarrow} \pi_{i+n} \Sigma^{n} X \stackrel{H}{\rightarrow} \pi_{i} e_{n}^{2} X \stackrel{P}{\rightarrow} \pi_{i-1} X \rightarrow \ldots
$$

Since $n$ is arbitrary, this relates the "metastable" homotopy group $\pi_{i} X$ to the stable homotopy group $\pi_{i}^{s} X$. Since $\bigcup_{n}(2)$ is $\Sigma_{2}$-equivariantly equivalent to $S^{n-1}, e_{n}^{2} X$ is equivalent to the quadratic construction $S^{n-1} \ltimes_{\Sigma_{2}}(X \wedge X)$. The latter space is homeomorphic to $\Sigma^{r}\left(R P^{n+r-1} / R P^{r-1}\right)$ when $X=S^{r}$, and the classical $E H P$-sequence is obtained by setting $n=1$.

The second application is a decomposition of the spectrum $Q_{\infty} C_{n} X$ into the wedge over $j \geqslant 1$ of the spectra $Q_{\infty} e_{n}^{j} X$ when $X$ is connected.

We allow $n=\infty$ in the approximation theorem, in which case $\Omega^{\infty} \Sigma^{\infty} X$ is to be interpreted as $Q X$. In particular, we have a group completion

$$
\alpha_{\infty}: \amalg K\left(\Sigma_{j}, 1\right)=C_{\infty} S^{0} \rightarrow Q S^{0} .
$$

We shall obtain successive elaborations of this homological comparison between symmetric groups and the stable homotopy groups of spheres.

Notes. For connected spaces, the approximation theorem was proven in $[45, \S \S 6,7]$. The general case was later proven geometrically by Segal [66] and by explicit computation of the relevant Pontryagin rings by Cohen [20]. The case $n=\infty$ is much simpler than the case $n<\infty$; it was implicit in Dyer and Lashof [22] and was explicit in the unpublished preprint version of their paper. The present form of the EHP sequence was derived by Milgram [52] from his earlier combinatorial cellular models for $\Omega^{n} \Sigma^{n} X$ [50]. The splitting of $Q_{\infty} C_{n} X$ is due to Snaith [69], the splitting in the case $n=\infty$ having been obtained earlier by Kahn [32].

5. The recognition principle. The black box in our schematic picture is a machine for the construction of spectra from $E_{\infty}$ spaces. In other words we can recognize spectra in the guise of spaces equipped with appropriate internal structure. Stripped to basics, the assertion is that if $X$ is an $E_{\infty}$ space, then there is a spectrum $\mathbf{B} X=\left\{B_{i} X\right\}$ and an $E_{\infty}$ map $\iota: X \rightarrow B_{0} X$ which is a group completion. We shall write $B_{0} X=\Gamma X$ as a reminder of the group completion property. Since $\pi_{0} X$ need not be a group, inverses not having been built into operad actions, we cannot expect $X$ to be equivalent to $\Gamma X$ in general.

While we do not wish to go into detail, a little discussion of the black box may be illuminating, especially as the assertion that $\iota: X \rightarrow \Gamma X$ is an $E_{\infty}$ map has no obvious meaning: we have insisted that an $E_{\infty}$ space $X$ can be a $\mathcal{C}^{\prime}$-space for any $E_{\infty}$ operad $\mathcal{C}^{\prime}$, whereas $\Gamma X$, as an infinite loop space, is naturally a $\mathcal{C}_{\infty}$-space. We set $\mathcal{C}=\mathcal{C}_{\infty} \times \mathcal{C}^{\prime}$ and observe that, by pullback along the projections, both $X$ and $\Gamma X$ are $\mathcal{C}$-spaces. (More generally, we can start over with $X$ assumed given as a $\mathcal{C}$-space.) When a monad $C$ acts (from the right) on a functor $F$ and acts on a space $X$, there is a very natural resulting space, the two-sided bar construction $B(F, C, X)$. The maps $\alpha_{n}$ : $C_{n} X \rightarrow \Omega^{n} \Sigma^{n} X$ give maps $\Sigma^{n} C_{n} X \rightarrow \Sigma^{n} X$ by adjunction, and the latter maps 
specify an action of $C_{n}$ on $\Sigma^{n}$. Therefore $C_{n} \times C^{\prime}$ also acts on $\Sigma^{n}$ (again, via the projection). The $i$ th space of the spectrum $\mathbf{B} X$ is specified by

$$
B_{i} X=\text { ind } \lim \Omega^{j} B\left(\Sigma^{i+j}, C_{i+j} \times C^{\prime}, X\right) .
$$

The full statement of the recognition principle asserts that there is a natural diagram of $\mathcal{C}$-maps

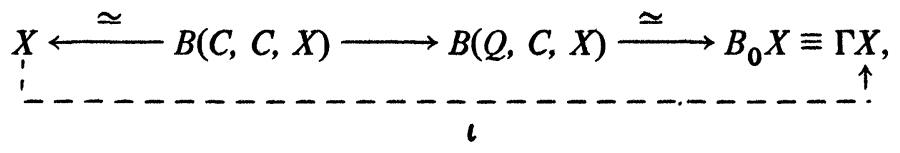

the first and third arrows of which are equivalences; that the middle arrow is a group completion is a consequence of the case $n=\infty$ of the approximation theorem. The map $\iota$ is the displayed composite of $\mathcal{C}$-maps and a homotopy inverse of a $C$-map which is an equivalence. It is in this sense that $\iota$ is an $E_{\infty}$ map. In effect then, our topological constructions have naturally converted the given $C^{\prime}$ geometry on $X$ to the canonical little cubes geometry on $\Gamma X$.

Looking back at our schematic picture, we see that there are several obvious consistency questions to ask. If we start with a spectrum $E$, regard its zeroth space as a $\bigodot_{\infty}$-space (and thus a $\bigodot_{\infty} \times \bigodot^{\prime}$-space for any $e^{\prime}$ ), and apply the black box, we obtain a new spectrum $\mathbf{B} E_{0}$. We expect some relationship between $E$ and $\mathbf{B} E_{0}$, and in fact the interior of the black box gives a natural map $\omega: \mathbf{B} E_{0} \rightarrow E$ of spectra whose zeroth map $\Gamma E_{0} \rightarrow E_{0}$ is an equivalence.

Again, given a space $X$, the interior of the black box quite formally gives a natural equivalence of spectra $\mathbf{B} C X \rightarrow Q_{\infty} X$. In particular, with $X=S^{0}$, we have

$$
\mathbf{B}\left(\coprod K\left(\Sigma_{j}, 1\right)\right)=\mathbf{B} C S^{0} \simeq Q_{\infty} S^{0} .
$$

In other words, the sphere spectrum can be constructed out of symmetric groups. This statement is less well understood than its homological counterpart of the previous section. For example, we have little idea as to how it might be used to obtain information about the stable homotopy groups of spheres.

NotES. See $[45, \S 14]$ and $[46, \S 2]$ or $[48, \mathrm{VII}]$ for details. There are many other black boxes. The first was constructed by Boardman and Vogt [13], [14], and their work was the essential starting point for all that followed. The second was constructed by Segal [67], and he was the first to consider applications to the classifying spaces of categories with appropriate internal structure (see \$8). Others were constructed by Anderson [4] and by Barratt and Eccles [11]. At the present time it is not known whether or not the spectra produced by different black boxes from the same input are equivalent, and much of the material below will depend on special features of our particular black box. The equivalence between $\Gamma\left(\amalg K\left(\Sigma_{j}, 1\right)\right)$ and $Q S^{0}$ is known as the Barratt-Quillen theorem since versions of this result were proven by Barratt [10], [11] and Quillen [unpublished, 67] independently.

6. Homotopy invariance of the recognition principle. We allowed ourselves to think of a homotopy inverse of an $E_{\infty}$ map which was an equivalence as an $E_{\infty}$ map in the previous section. Clearly there ought to be a more general notion of $\mathcal{C}$-map between $\mathcal{C}$-spaces which accepts such homotopy inverses. 
Again, there clearly ought to be a generalized notion of e-space which is invariant under homotopy equivalence. Lada [20] has developed an appropriate theory, and we give a quick sketch of it here.

An action $\theta: C X \rightarrow X$ of a monad on a space is analogous to an action $G \times X \rightarrow X$ of a monoid on a space. It is well understood how to study the latter type of action up to homotopy, or rather up to all higher associativity homotopies, this being only a slight generalization of Stasheff's theory of $A_{\infty}$ spaces [71]. One begins with a map $G \times X \rightarrow X$ such that the operations $g_{1}\left(g_{2} x\right)$ and $\left(g_{1} g_{2}\right) x$ are homotopic. This yields two homotopies between the operations $\left(g_{1}\left(g_{2}\left(g_{3} x\right)\right)\right)$ and $\left(g_{1} g_{2} g_{3}\right) x$, and one wants these homotopies to be homotopic, and so on. Generalizing this idea to monad actions, one arrives at the notion of an sh $C$-space (sh = strong homotopy).

Using a variant of the bar construction $B(C, C, X)$, one then constructs an actual $C$-space $U X$ equivalent to any given sh $C$-space $X$ and defines an "SH $C$-map" $f: X \rightarrow Y$ between sh $C$-spaces $X$ and $Y$ to be an actual $C$-map $U X \rightarrow U Y$ (to be thought of as the underlying map $X \simeq U X \rightarrow U Y \simeq Y$ with extra structure). With the obvious composition, there results a category of sh $C$-spaces and SH $C$-maps, and there is also an obvious homotopy category. The generalization of the recognition principle to this category is an immediate application of the recognition principle for actual $\mathcal{C}$-spaces already sketched.

The basic homotopy invariance statements are as follows.

(1) If $Y$ is an sh $C$-space and $f: X \rightarrow Y$ is an equivalence, then $X$ is an sh $C$-space and $f$ is an SH $C$-map.

(2) If $f: X \rightarrow Y$ is an SH $C$-map between sh $C$-spaces and $g \simeq f$, then $g$ is an SH $C$-map and $g \simeq f$ as SH $C$-maps.

(3) If $f: X \rightarrow Y$ is an SH $C$-map between sh $C$-spaces which is an equivalence with homotopy inverse $g$, then $g$ is an SH $C$-map and $f g=1$ and $g f \simeq 1$ as SH $C$-maps.

The basic idea of the proof is to define a notion of sh $C$-map $X \rightarrow Y$, using higher homotopies, when one of $X$ and $Y$ is a $C$-space and the other is an sh $C$-space (this being much simpler technically than the case when both $X$ and $Y$ are sh $C$-spaces), to prove strong homotopy analogs of special cases of (1)-(3) by direct manipulation of higher homotopies, and to then deduce (1)-(3) by using properties of the construction $U X$ to relate sh $C$-maps to $\mathrm{SH}$ C-maps.

Notes. The original black box of Boardman and Vogt [13], [14] was also homotopy invariant, this being one of its most celebrated features. Indeed, in their approach, analysis of homotopy invariance necessarily preceded the recognition principle. They made room for higher homotopies by blowing up the given operad rather than by blowing up the spaces on which the operad acts.

7. $\mathcal{G}_{*}$ functors and classifying spaces; $\mathcal{L}$. Consider the category $\mathcal{G}_{*}$ of finite dimensional real inner product spaces and linear isometric isomorphisms. There are lots of functors $G: \mathscr{G}_{*} \rightarrow \mathcal{T}$ which admit commutative and associative natural sum operations $\omega: G V \times G W \rightarrow G(V \oplus W)$. We refer to such $G$ as $G_{*}$ functors. The most obvious example is given by the orthogonal groups OV. 
We claim that $g_{*}$ functors naturally give rise to $E_{\infty}$ spaces. The relevant $E_{\infty}$ operad is the linear isometries operad $\mathcal{L}$. With $R^{\infty}=$ ind $\lim R^{n}$ given its natural inner product, $\mathscr{L}(j)$ is the space of linear isometries $\left(R^{\infty}\right)^{j} \rightarrow R^{\infty}$, $\left(R^{\infty}\right)^{j}$ being the direct sum of $j$ copies of $R^{\infty}$. By passage to limits, it is easy to extend an $g_{*}$ functor $G$ to a functor $G$ and natural sum $\omega$ defined on finite or countably infinite dimensional real inner product spaces and their linear isometries. The $\mathcal{L}$-space associated to $G$ is $G R^{\infty}$; its $\mathcal{L}$-action $\theta$ is specified by

$$
\theta_{j}\left(f, g_{1}, \ldots, g_{j}\right)=(G f)\left(g_{1} \oplus \cdots \oplus g_{j}\right)
$$

for $f \in \mathcal{L}(j)$ and $g_{i} \in G R^{\infty}$. By abuse, we shall abbreviate $G R^{\infty}=G$.

The various classical groups and their homogeneous and classifying spaces occur in nature as $Q_{*}$ functors and thus determine $P_{-}$-spaces and associated spectra. Certain of these spaces $X$ also appear in the periodic spectra given by the Bott periodicity theorem, and in these cases $\mathbf{B} X$ is equivalent to the connective spectrum obtained from the relevant Bott spectrum by killing its homotopy groups in negative degrees. Indeed, the Bott maps all arise from maps of $g_{*}$ functors of the form $H / H_{1} \times H_{2} \rightarrow \Omega\left(G / G_{1} \times G_{2}\right)$, and the assertion follows from a consistency statement of the form $\Omega \mathbf{B} X \simeq \mathbf{B} \Omega X$ coming from the interior of our black box together with a general characterization of the connective spectrum associated to a periodic spectrum.

Perhaps the most interesting $\mathscr{G}_{*}$ functor is $\tilde{F}$, where $\tilde{F} V$ is the function space $F(t V, t V)$ of based maps from the one point compactification of $V$, denoted $t V$, to itself. The sum $\omega$ is given by the smash product of maps via $t V \wedge t W \cong t(V \oplus W)$. Write

$$
\Omega^{v} X=F(t V, X) \text { and } \Sigma^{v} X=X \wedge \dot{t} V
$$

for the loop and suspension functors determined by the sphere $t V$ and note that

$$
\tilde{F} \equiv \tilde{F} R^{\infty}=\text { ind } \lim \Omega^{v} t V \cong \text { ind } \lim \Omega^{v} \Sigma^{v}\left(S^{0}\right) \cong Q S^{0} .
$$

Thus the $\mathcal{L}$-space $\tilde{F}$ is also the zeroth space of the sphere spectrum $Q_{\infty} S^{0}$, this being the starting point for the analysis of multiplicative structure on spectra.

$\tilde{F}$ contains the sub $g_{*}$ functors $F$, Top, and $O$ of homotopy equivalences, homeomorphisms, and (compactifications of) orthogonal transformations; restriction to degree one maps yields the further sub ${ }_{*}$ functors $S F, S$ Top, and $S O$. Each of these is in fact an ${ }_{*}$ monoid, in the sense that composition of maps $G V \times G V \rightarrow G V$ specifies a morphism of $g_{*}$ functors $G \times G \rightarrow G$. It follows that application of the standard product-preserving classifying space functor $B$ yields a new ${ }{ }_{*}$ functor $B G$, and of course the spaces $B G V$ classify the appropriate types of bundles and fibrations. On the infinite loop space level, another consistency statement coming from the interior of our black box gives that $B G$ and the machine-built delooping $B_{1} G$ of $G$ are equivalent in the sense that $\mathbf{B}(B G)$ and $\mathbf{B}\left(B_{1} G\right)$ are equivalent spectra. In particular, $B G$ and $B_{1} G$ are equivalent spaces, and both classify stable bundles and fibrations of the appropriate type.

PL-bundles do not fit readily into this picture, but the triangulation theorem obviates any need for an elaborate theory: $B \mathrm{PL}$ is the fibre of $B$ Top $\rightarrow K\left(Z_{2}, 4\right)$ as an infinite loop space. 
Notes. See [48, I] for details. The invention of the linear isometries operad and the passage from $G_{*}$ functors to $E_{\infty}$ spaces are due to Boardman and Vogt [13], [14], this being the motivating example for their original theory.

8. Symmetric monoidal and permutative categories; $\mathscr{D}$. Categories with suitable internal structure provide another rich source of $E_{\infty}$ spaces and thus of spectra. In particular, infinite loop spaces whose homotopy groups are the algebraic $K$-groups of rings arise in this way from the direct sum on the categories of projective modules and their isomorphisms.

A symmetric monoidal category $(\mathbb{Q}, \square, *)$ consists of a (small, topological) category $\mathbb{Q}$, a functor $\square: \mathbb{Q} \times \mathbb{Q} \rightarrow \mathbb{Q}$, an object $*$, and coherent natural isomorphisms

$$
\begin{aligned}
a: A \square(B \square C) & \cong(A \square B) \square C, \\
b: * \square A & \cong A, \quad \text { and } c: A \square B \cong B \square A .
\end{aligned}
$$

Here coherence means that all diagrams which can reasonably be expected to commute do in fact commute. (See Mac Lane [37, \$15] for an intuitive discussion of this concept.) Such categories occur ubiquitously in nature. $c^{\prime}$ ' is said to be permutative if $a$ and $b$ (but emphatically not $c$ ) are identity maps. Such categories occur less commonly, but there is a natural way to replace symmetric monoidal categories by equivalent permutative categories.

There is a standard product-preserving classifying space functor $B$ from categories to spaces. (A category with a single object is a monoid, and here $B$ coincides with the classifying space functor of the previous section.) We claim that $B$ takes permutative categories to $E_{\infty}$ spaces. The relevant $E_{\infty}$ operad is called $\mathscr{D}$. Let $\tilde{\Sigma}_{j}$ denote the category whose objects are the elements of $\Sigma_{j}$ and whose morphisms are elements of $\Sigma_{j}$ regarded as translations $\tau: \sigma \rightarrow \sigma \tau \cdot \mathscr{D}(j)$ is defined to be $B \tilde{\Sigma}_{j}$, and the structural maps $\gamma$ of $\mathscr{D}$ are obtained by applying $B$ to appropriate functors $\tilde{\gamma}$. If $Q$ is a permutative category, then $c$ and $\square$ determine functors $\tilde{\Sigma}_{j} \times \mathbb{Q}^{j} \rightarrow \mathbb{Q}$. Application of $B$ to these functors yields an action $\theta$ of $\mathscr{D}$ on $B$ Q .

A few words should be said about the functoriality of $B$. A map $f: \Delta \mathbb{C}^{\prime} \rightarrow\left(\mathrm{C}^{\prime}\right)$ of permutative categories is required to preserve $\square, *$, and $c ; B f: B\left(\mathbb{P}^{*} \rightarrow\right.$ $B Q^{\prime}$ is then a map of $\mathscr{D}$-spaces. One could weaken the conditions on $f$ so as to allow $f(A \square B)$ to be coherently naturally isomorphic to $f A \square f B$. Bf is then an SH $D$-map since the functoriality properties of the replacement of symmetric monoidal categories by permutative categories give that $B f$ is the composite of a $D$-map and a homotopy inverse of a $D$-map which is an equivalence.

If $A$ is a (topological, but perhaps discrete) ring, there is a permutative category $\mathcal{G} \mathfrak{L A}$ whose objects are the natural numbers and whose morphisms $n \rightarrow n$ are the elements of the general linear group $\mathrm{GL}(n, A)$. (There are no morphisms $m \rightarrow n$ if $m \neq n$.) The product on $\mathcal{G} \mathscr{L} A$ is given by the direct sum of matrices. When $A$ is discrete, it is a direct consequence of the group completion property that the component of the basepoint of $\Gamma B Q E A$ has the same homotopy type as Quillen's plus construction on $B \mathrm{GL}(\infty, A)$ [63], [64]. Indeed, the plus construction is the least structured and the black box the most structured way of obtaining this homotopy type. It follows that Quillen's algebraic $K$-groups of $A$ are given by 


$$
K_{i} A=\pi_{i} \Gamma B \mathcal{G} \mathcal{E} A \text { for } i>0 .
$$

Of course, the groups $K_{i} A$ have been most studied when $A$ is commutative. Here one naturally expects the tensor product of matrices to yield a multiplicative structure suitably related to the additive structure on $B Q E A$. We are inexorably led to consider spaces with two $E_{\infty}$ structures.

Notes. See [46, §4] and [48, VI, §§3-5] for details and further examp!es. The first passage from categories to spectra was due to Segal [67], and the relevance of permutative categories was noted by Anderson [4]. The passage from symmetric monoidal to permutative categories was originally due to Isbell [28].

\section{Chapter II. Multiplicative INFinite loop SPACE THEORY}

9. Operad pairs and their actions; $(\mathfrak{T}, \mathscr{N})$. We have finished drawing the additive left-hand side of our schematic picture. Its elaboration to a multiplicative picture amounts essentially to a change of ground categories from spaces to $E_{\infty}$ spaces. We describe the notion of an $E_{\infty}$ ring space here, the problem being to succinctly record all possible distributivity relations between an additive and a multiplicative $E_{\infty}$ structure on a space.

Fix an operad $\mathcal{G}$. Actions by $\mathcal{G}$ are to be thought of as multiplicative, and we define a $\mathcal{G}_{0}$-space, or $\mathcal{G}$-space with zero, to be a $\mathcal{G}$-space $X$ with action $\xi$ and basepoint 1 and with an element 0 such that $\xi_{j}\left(g, x_{1}, \ldots, x_{j}\right)=0$ if any $x_{i}=0$. We think of the category $\mathcal{G}_{0}[\mathcal{T}]$ of $\mathcal{G}_{0}$-spaces as our ground category.

Now let $\mathcal{C}$ be another operad. An action of $\mathcal{G}$ on $\mathcal{E}$ is a collection of maps

$$
\lambda: \mathcal{G}(k) \times \mathfrak{C}\left(j_{1}\right) \times \cdots \times \mathfrak{e}\left(j_{k}\right) \rightarrow \mathfrak{E}\left(j_{1} \cdots j_{k}\right)
$$

subject to certain axioms. These axioms serve to ensure that $(C, \mu, \eta)$ restricts to a monad in the category $\mathcal{G}_{0}[\mathcal{T}]$. That is, if $X$ is a $\mathcal{S}_{0}$-space, then $C X$ is again a $\mathcal{G}_{0}$-space and $\mu: C C X \rightarrow C X$ and $\eta: X \rightarrow C X$ are maps of $\mathcal{G}_{0}$-spaces. To make this a little less mysterious, we describe how the action $\xi$ of $\mathcal{\xi}$ on $X$ is to induce an action (again denoted $\xi$ ) of $\mathcal{G}$ on $C X$. In fact, the requisite maps $\xi_{k}$ : $\mathcal{G}(k) \times(C X)^{j} \rightarrow C X$ result by passage to quotient spaces from the maps

$$
\xi_{k}: \mathcal{G}(k) \times \mathcal{C}\left(j_{1}\right) \times X^{j_{1}} \times \cdots \times \mathcal{C}\left(j_{k}\right) \times X^{j_{k}} \rightarrow \mathfrak{E}\left(j_{1} \cdots j_{k}\right) \times X^{j_{1}} \cdots j_{k}
$$

specified by

$$
\xi_{k}\left(g, c_{1}, y_{1}, \ldots, c_{k}, y_{k}\right)=\left(\lambda\left(g, c_{1}, \ldots, c_{k}\right), \times_{l} \xi_{k}\left(g, y_{l}\right)\right),
$$

where $I$ runs over the set of all sequences $\left(i_{1}, \ldots, i_{k}\right)$ with $1 \leqslant i_{r} \leqslant j_{r}$, ordered lexicographically, and where

$$
y_{I}=\left(x_{1 i_{1}}, \ldots, x_{k i_{k}}\right) \in X^{k} \text { if } y_{r}=\left(x_{r 1}, \ldots, x_{r j}\right) \in X^{j_{r}} \text {. }
$$

When $\mathcal{G}$ acts on $\mathcal{C}$, we refer to $(\mathcal{C}, \mathcal{G})$ as an operad pair. Define a $(\mathfrak{C}$. $\mathcal{G})$-space to be a $C$-space in the category $\mathcal{G}_{0}[\widetilde{T}]$. Thus a $(\mathcal{E}, \mathcal{E})$-space $(X, \theta, \xi)$ is a $\mathcal{G}_{0}$-space $(X, \xi)$ and a $\mathcal{C}_{\text {-space }}(X, \theta)$ such that $\theta: C X \rightarrow X$ is a map of $\mathcal{G}$-spaces (and thus of $\mathcal{G}_{0}$-spaces since $\theta(0)=0$ follows). An $E_{\infty}$ ring space is a $(\mathcal{C}, \mathcal{G})$-space where both $\mathcal{C}$ and $\mathcal{G}$ are $E_{\infty}$ operads.

To clarify the definitions, we consider a simple special case. Recall the operad $\mathscr{T}$ with each $\Re(j)$ a point. $\mathscr{N}$ acts on itself, the maps $\lambda$ being the only ones possible. An $(\mathscr{T}, \mathscr{K})$-space is a commutative semiring, all of the 
axioms for a commutative ring except the existence of additive inverses having been built in. Indeed, looking at the definition of the maps $\xi_{k}$ above, we see that $\theta: N X \rightarrow X$ being a map of $\mathfrak{X}$-spaces implies the general distributivity law

$$
\underset{r=1}{\stackrel{\times}{\times}}\left(\sum_{i=1}^{j_{r}} x_{r i}\right)=\sum_{I}\left(x_{1 i_{1}} \cdots x_{k i_{k}}\right) .
$$

The nullity of zero was built in by the defintion of an $\Re_{0}$-space.

Of course, for an operad pair $(\mathcal{C}, \mathcal{G}), C X$ is the free $(\mathcal{C}, \mathcal{G})$-space generated by a $\mathscr{G}_{0}$-space $X$. There is an obvious structure of $\mathcal{G}_{0}$-space on $S^{0}=\{0,1\}$ for any $\mathcal{G}$, and $S^{0} \rightarrow X$ is a map of $\mathcal{G}_{0}$-spaces for any $\mathcal{G}_{0}$-space $X$. Thus any (E), $\mathcal{G}$ )-space $X$ admits a unit $e: C S^{0} \rightarrow X$ which is a map of (e, $\left.\mathcal{G}\right)$-spaces.

Notes. See [48, VI, §§1-2] for details.

10. Symmetric bimonoidal and bipermutative categories; $(\mathscr{D}, \mathscr{D})$. A symmetric bimonoidal category $(\mathbb{Q}, \oplus, 0, \otimes, 1)$ is a pair of symmetric monoidal structures on a category $\Theta$ (with natural isomorphisms $a, b, c$ for $\oplus$ and $\tilde{a}, \tilde{b}$, $\tilde{\boldsymbol{c}}$ for $\otimes)$ and coherent natural isomorphisms

$$
d:(A \oplus B) \otimes C \cong(A \otimes C) \oplus(B \otimes C) \text { and } n: 0 \otimes A \cong 0 .
$$

(See Laplaza [34] for a thorough study of coherence in this context.) Such categories occur ubiquitously in nature. $\mathscr{Q}$ is said to be bipermutative if $a, b$, $\tilde{a}, \tilde{b}, d$, and $n$ (but not $c$ and $\tilde{c}$ ) are identity maps. Such categories occur less commonly, but there is a natural way to replace symmetric bimonoidal categories by equivalent bipermutative categories.

The $E_{\infty}$ operad $\mathscr{D}$ acts on itself, the requisite maps $\lambda$ being obtained by application of $B$ to appropriate functors $\tilde{\lambda}$. If $Q$ is a bipermutative category, $\theta$ is the action of $\mathscr{D}$ on $B Q$ derived from $\oplus$, and $\xi$ is the action of $\mathscr{D}$ on $B Q$ derived from $\otimes$, then $(B Q, \theta, \xi)$ is a $(\mathscr{D}, \mathscr{D})$-space. In our schematic picture, we now have the functor $B$ from bipermutative categories to $E_{\infty}$ ring spaces.

If $A$ is a commutative ring, then $\mathcal{G} \mathscr{L} A$ and its subcategory $\mathscr{C} A$ of orthogonal matrices are bipermutative categories. Another example is $\mathcal{E}$, the category with objects the natural numbers and with morphisms $\Sigma_{n}: n \rightarrow n$. $\mathcal{E}$ is to be thought of as (a skeleton of) the category of finite sets and their isomorphisms; disjoint union and Cartesian product give $\oplus$ and $\otimes . B \mathcal{E}$ may be identified with $D S^{0}$. For any bipermutative category $Q$, the unit $e: D S^{0} \rightarrow$ $B \mathscr{Q}$ arises by application of $B$ to a unit functor $\mathcal{E} \rightarrow \mathbb{Q}$. When $\mathbb{Q}=\mathcal{G} \mathscr{E} A$, this functor sends a permutation to the corresponding permutation matrix.

Notes. See [48, VI, §§3-5] for details and further examples.

11. $E_{\infty}$ ring spectra; $\left(\mathcal{K}_{\infty}, \mathfrak{e}\right)$. So far the passage from the additive to the multiplicative theory has been quite automatic. We now ask ourselves what sort of object the black box should spew out when fed in an $E_{\infty}$ ring space, and find the answer not at all obvious. We have one example in front of us, namely $Q_{\infty} S^{0}$, and the $\mathcal{L}$-space structure on its zeroth space surely ought to be part of its multiplicative structure. To see the full structure present it is convenient to modify our definition of a spectrum. Thus redefine a spectrum $E$ to consist of a space $E V$ for each finite dimensional subinner product space $V$ of $R^{\infty}$ such that $E V$ is homeomorphic to $\Omega^{w} E(V+W)$ whenever $W$ is orthogonal to $V$. Such a coordinate-free spectrum determines a sequential, or 
coordinatized, spectrum $\left\{E_{i}\right\}$ by $E_{i}=E R^{i}$, and it is not much more difficult to construct a coordinate-free spectrum from a coordinatized one.

Let $\mathcal{G}$ be any $E_{\infty}$ operad which maps onto $\mathcal{E}$, for example $E$ itself or $\mathfrak{L} \times \mathcal{G}^{\prime}$ for any $E_{\infty}$ operad $\mathcal{G}^{\prime}$. A $\mathcal{G}$-spectrum is, roughly, a spectrum $E$ together with an action of $\mathcal{G}$ on $E$ given by maps " $\xi_{j}: \mathcal{G}(j) \times E^{j} \rightarrow E$ ". More precisely, we require maps

$$
\xi_{j}(g): E V_{1} \wedge \cdots \wedge E V_{j} \rightarrow E g\left(V_{1} \oplus \cdots \oplus V_{j}\right)
$$

for each $g \in \mathcal{G}(j)$, where $g$ is regarded as a linear isometry $\left(R^{\infty}\right)^{j} \rightarrow R^{\infty}$ via the map $\mathcal{G}(j) \rightarrow \mathscr{L}(j)$. The maps $\xi_{j}(g)$ are subject to a number of axioms. These imply, in particular, that $E_{0}$ is a $\mathcal{G}_{0}$-space, and it should be admitted that we do not yet fully understand how to exploit the rest of the structure present. An $E_{\infty}$ ring spectrum is a $\mathcal{G}$-spectrum for any $E_{\infty}$ operad $\mathcal{G}$ over $\mathscr{E}$.

The homotopy groups of the zeroth space $E_{0}$ of a $\mathcal{G}$-spectrum $E$ form a commutative ring (in the graded sense: $x y=(-1)^{\operatorname{deg} y \operatorname{deg} x} y x$ ). They also admit various homotopy operations, the analysis of which has only just begun. For example, the map $\xi_{2}: \mathcal{G}(2) \ltimes_{\Sigma_{2}} E_{0} \wedge E_{0} \rightarrow E_{0}$ allows the construction of $\cup_{i}$-products in $\pi_{*} E_{0}$ which generalize the known operations in $\pi_{*} Q S^{0}$ (Kahn [30], Milgram [53]).

In line with our general proposition that the multiplicative theory results from the additive theory by a change of ground categories, $Q_{\infty} X$ is a $\mathcal{G}$-spectrum whenever $X$ is a $\mathcal{G}_{0}$-space. Moreover, if $E$ is a $\mathcal{G}$-spectrum, a map $X \rightarrow E_{0}$ of $\mathcal{G}_{0}$-spaces uniquely determines a map $Q_{\infty} X \rightarrow E$ of $\mathcal{G}$-spectra. In particular, $E$ has a unit $e: Q_{\infty} S^{0} \rightarrow E$ which is a map of $\mathcal{G}$-spectra. As we shall see, $e$ plays a vital role in the applications.

Looking at our schematic picture, we see that our description of $E_{\infty}$ ring spectra appears to be incomplete. Their zeroth spaces ought to be $E_{\infty}$ ring spaces in order to have the dotted arrow from $E_{\infty}$ ring spectra to $E_{\infty}$ ring spaces. Now both the infinite little cubes operad $\mathcal{C}_{\infty}$ and $\mathcal{G}$ act on $E_{0}$ if $E$ is a $\mathcal{G}$-spectrum, but there is clearly no way to make $\mathcal{G}$ act on $\mathcal{E}_{\infty}$ : cubes are manifestly not invariant under orthogonal transformations. We require a new operad which behaves like $\mathcal{C}_{\infty}$ in the additive theory but which is acted upon by $\mathcal{L}$ and thus also by $\mathcal{G}$.

We are led to define the little convex bodies operad $\mathcal{K}_{\mathfrak{t}}$ for a finite dimensional real inner product space $V$. The points of $\mathcal{K}_{v}(j)$ are $j$-tuples of little convex bodies with disjoint images. Here little convex bodies are appropriate embeddings $V \rightarrow V$. The precise definition need not concern us, but the essential fact is that if $f: V \rightarrow W$ is a linear isometric isomorphism and $k: V \rightarrow V$ is a little convex body, then $f k f^{-1}: W \rightarrow W$ is a little convex body. $\mathcal{K}_{v}$ acts naturally on $\Omega^{v} X$ for any space $X$. By passage to limits over $V \subset R^{\infty}$, there results an $E_{\infty}$ operad $\mathcal{K}_{\infty}$ which acts naturally on infinite loop spaces. $\mathcal{L}$ acts on $\mathcal{K}_{\infty}$, and the zeroth space of a $\mathcal{G}$-spectrum is a ( $\mathfrak{K}_{\infty}$, $\mathcal{G})$-space. We have the dotted arrow from $E_{\infty}$ ring spectra to $E_{\infty}$ ring spaces.

NoTES. See [48, IV, $\$ 1$ and VII, $\S \S 1,2]$ for details. The invention of $E_{\infty}$ ring spectra was joint work with Frank Quinn and Nigel Ray. The description of the $\mathcal{K}_{v}$ here is not quite accurate, since they are not quite operads, but suppression of the relevant technical problems gives a clearer picture of the essentials of the theory. 
12. The recognition principle. To complete the discussion of our schematic picture, we reconsider the black box in the context of $E_{\infty}$ ring spaces. The basic assertion is that if $X$ is an $E_{\infty}$ ring space, then $\mathbf{B} X$ is an $E_{\infty}$ ring spectrum and the natural group completion $\iota: X \rightarrow \Gamma X$ is a map of $E_{\infty}$ ring spaces.

Again, a little discussion may prove illuminating. Given a pair ( $\left.\mathfrak{C}^{\prime}, G^{\prime}\right)$ of $E_{\infty}$ operads, for example $(\mathscr{D}, \mathscr{D})$, we set $\left(\mathcal{E}^{\prime}, \mathcal{Q}\right)=\left(\mathscr{H}_{\infty}^{\circ} \times \mathfrak{E}^{\prime}, E^{\prime} \times \vec{G}^{\prime}\right)$. Given a $(\mathcal{C}, \mathcal{G})$-space, for example a $\left(\mathcal{C}^{\prime}, \mathcal{G}^{\prime}\right)$-space, we construct the spectrum $\mathbf{B} X$ out of the additive $E_{\infty}$ structure. Here we work in the coordinate-free setting, with little convex bodies, and set

$$
(B V)(X)=\text { ind } \lim _{W \perp V} \Omega^{w} B\left(\Sigma^{c+n^{\prime}}, K_{c+w} \times C^{\prime}, X\right) .
$$

The full multiplicative elaboration of the recognition principle asserts that all maps (including $\iota$ ) are $\mathcal{G}$-maps in the diagram

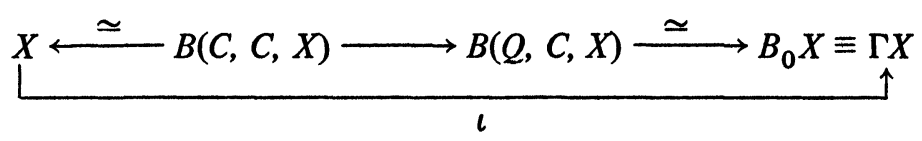

In effect, since the middle arrow and thus $\iota$ are group completions, we have group completed the additive $E_{\infty}$ structure while carrying along the multiplicative $E_{\infty}$ structure.

If $E$ is a $\mathcal{G}$-spectrum, then the natural map $\omega: \mathbf{B} E_{0} \rightarrow E_{0}$ is a map of $\mathcal{G}$-spectra. If $X$ is a $\mathcal{G}_{0}$-space, then the natural equivalence $\mathbf{B} C X \rightarrow Q_{\infty} X$ is a map of $\mathcal{G}$-spectra. In particular, with $X=S^{0}$,

$$
\mathbf{B}\left(\amalg K\left(\Sigma_{j}, 1\right)\right)=\mathbf{B} C S^{0} \simeq Q_{\infty} S^{0}
$$

as an $E_{\infty}$ ring spectrum, this being the most structured version of the Barratt-Quillen theorem. It implies an equivalence of $\hat{S}$-spaces between $\Gamma C S^{0}$ and $\tilde{F}$ and therefore implies that the infinite loop structure on $F$, and thus also on the classifying space $B F$ for stable spherical fibrations, can be constructed out of symmetric groups.

With $X=B \mathcal{Q} \mathcal{L} A$ for a commutative ring $A$, we have given the spectra of algebraic $K$-theory an $E_{\infty}$ ring structure. In particular, $K_{*} A$ is a commutative ring and the unit $e: Q S^{0} \rightarrow \Gamma B \mathcal{Q} \mathcal{E A}$ induces a map of rings $\pi_{*}^{s} \rightarrow K_{*} A$. Much of the little that is presently known about $K_{*} Z$ comes out of analysis of this map.

We have so far been discussing application of the one operad recognition principle to the additive $E_{\infty}$ structure $(X, \theta)$ of a $(\mathcal{Q}, \mathcal{G})$-space $(X, \theta, \xi)$. It is natural to ask what can be said about application of the one operad recognition principle to the multiplicative $E_{\infty}$ structure $(X, \xi)$. In fact, the resulting spectrum is trivial because of the presence of the zero component of $X$. However, suppose that $\pi_{0} X$ is the nonnegative integers (as holds when $X=B \mathcal{E}$ or $X=B \mathcal{Q} \mathcal{E} A$ for example) and let $M$ be a nontrivial multiplicative submonoid of the positive integers. Let $X_{M}$ be the union of the corresponding components of $X$. Then $X_{M}$ is a sub $\mathcal{G}$-space of $X$. If $\Gamma_{1}\left(X_{M}, \xi\right)$ and $\Gamma_{1}(X, \theta)$ denote the 1-components of the zeroth spaces of the spectra associated to $\left(X_{M}, \xi\right)$ and $(X, \theta)$, then there is a map $\Gamma_{1}(X, \theta) \rightarrow \Gamma_{1}\left(X_{M}, \xi\right)$ of multiplicative $E_{\infty}$ spaces which is a localization at $M$. In other words, 
although $\Gamma_{1}(X, \theta)$ is constructed by use of the additive $E_{\infty}$ structure, its localizations depend only on the multiplicative $E_{\infty}$ structure.

Notes. See [48, VII, §§4, 5] for details. Versions of two special cases of the assertion about $\Gamma_{1}\left(X_{M}, \xi\right)$.were proven by Tornehave [78], [79]. Homotopy invariance has not yet been studied in the $E_{\infty}$ ring context.

13. $\mathscr{I}_{*}$ prefunctors and Thom spectra. The notion of an $E_{\infty}$ ring spectrum may seem rather daunting. We indicate here how the various Thom spectra occur in nature as $E_{\infty}$ ring spectra, without recourse to the black box.

Just as the familiar submonoids of $F V$ give $\mathscr{G}_{*}$ functors, so their Thom spaces give $\mathscr{G}_{*}$ prefunctors. Just as $\mathscr{G}_{*}$ functors lead to $\mathcal{L}_{\text {-spaces, so }} \mathscr{G}_{*}$ prefunctors lead to $\mathcal{L}$-spectra. We proceed to construct a diagram

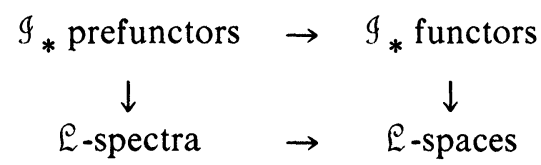

An $\mathscr{I}_{*}$ prefunctor is a functor $T: \mathscr{I}_{*} \rightarrow \mathcal{T}$, an associative and commutative natural Whitney sum map $\omega: T V \wedge T W \rightarrow T(V \oplus W)$, and a natural map $e$ : $t V \rightarrow T V$ such that the adjoints $T V \rightarrow \Omega^{w} T(V \oplus W)$ of the maps $\omega \circ(1 \wedge e)$ are closed inclusions. An obvious example is $T=t$, the one-point compactification functor on inner product spaces.

An $\mathcal{G}_{*}$ prefunctor $T$ determines an $\mathcal{G}_{*}$ functor $\tilde{F} T$ with $(\tilde{F} T)(V)=\Omega^{v} T V$. For example, $\tilde{F} t$ is the $g_{*}$ functor $\tilde{F}$ considered in $\$ 7$. An $g_{*}$ prefunctor $T$ determines an $\mathcal{L}$-spectrum $M T$ by $(M T)(V)=$ ind $\lim \Omega^{w} T(V+W)$. The requisite maps $\xi_{j}$ result from the maps $T f$ (for an isometry $f$ ) and the Whitney sum by passage to limits. For example, $M t$ is $Q_{\infty} S^{0}$. The zeroth space of $M T$ is precisely the $\mathcal{L}$-space associated to the $\mathcal{G}_{*}$ functor $\tilde{F} T$.

Now let $G$ be an $\mathscr{G}_{*}$ functor, or rather an $\mathcal{G}_{*}$ monoid, which maps to $F, j$ : $G \rightarrow F . B G V$ classifies " $G V$-bundles with fibre $t V$ and a canonical section". (By abuse, we refer to $G V$-bundles even when $G=F$, when they are only spherical fibrations.) The Thom space $T \xi$ of such a bundle $\xi$ is the quotient of the total space by the base-space. In the case of vector bundles, we are applying one-point compactification fibrewise and then identifying all the points at infinity. There is a canonical universal $G V$-bundle $p: E G V \rightarrow B G V$ with Thom space $T G V=E G V / B G V$. The inclusion of the fibre over the basepoint of $B G V$ gives $e: t V \rightarrow T G V$ and the bundle map over the natural map $B G V \times B G W \rightarrow B G(V \oplus W)$ gives $\omega: T G V \wedge T G W \rightarrow T G(V \oplus W)$. With these maps, $T G$ is an $\Phi_{*}$ prefunctor. $M G=M T G$ is the Thom spectrum of $G$.

Notes. See [48, IV, §2] for details. This material was joint work with Frank Quinn and Nigel Ray.

14. Orientation theory and $B(G ; E)$. Orientations of bundles with respect to cohomology theories play a central role in topology. In particular, they are vital to Adams' analysis of vector bundles [1] and to Sullivan's analysis of topological bundles [74].

As in the previous section, let $G$ be an $\Phi_{*}$ monoid with a map $j: G \rightarrow F$. Let $E$ be a ring spectrum. An $E$-orientation of a $G V$-bundle $\xi: Y \rightarrow X$, dim $V=n$, is a class $\mu \in E^{n} T \xi$ (i.e., a homotopy class $T \xi \rightarrow E V$ ), such that $i^{*} \mu$ 
generates $E^{*}\left(\xi^{-1} x\right)$ as a $\pi_{*} E$-module for each $x \in X$, where $i: \xi^{-1} x \rightarrow Y$ is the inclusion. At least if $X$ is a finite dimensional CW-complex, the cup product with $\mu$ then yields a Thom isomorphism $E^{*}\left(X^{+}\right) \rightarrow E^{*}(T \xi)$. (A disjoint basepoint is added to $X$ since reduced theories are understood.) If $\mu^{\prime}$ is a second orientation of $\xi$, then $\mu^{\prime}=\nu \mu$ for some unit $\nu$ in the ring $E^{0}\left(X^{+}\right)$.

Since $E$ is a ring spectrum, $\pi_{0} E_{0}$ is a ring. Let $F E$ denote the union of the unit components of $E_{0}$ and let $S F E$ denote the component of the identity. $F E$ classifies the units in $E^{0}\left(X^{+}\right)$. In particular, $F Q_{\infty} S^{0}=F$ and $S F Q_{\infty} S^{0}=$ $S F$, and the unit $e: Q_{\infty} S^{0} \rightarrow E$ of $E$ restricts to a map $e: F \rightarrow F E$.

There is a canonical classifying space $B(G V ; E)$ for $E$-oriented $G V$-bundles. Indeed, by virtue of our definition of spectra, the monoids $F V$ and thus also $G V$ act from the right on $F E$, and $B(G V ; E)$ is the two-sided bar construction $B(F E, G V, *)$. There is a fibration sequence (with ej the composite $G V \rightarrow F V \subset F \rightarrow F E$ )

$$
G V \stackrel{e j}{\rightarrow} F E \stackrel{\tau}{\rightarrow} B(G V ; E) \stackrel{q}{\rightarrow} B G V .
$$

Here $q$ corresponds to the forgetful functor from $E$-oriented $G V$-bundles to $G V$-bundles and $\tau$ corresponds to the functor which sends a unit to the trivial bundle oriented by the product of the unit and a certain canonical orientation of the trivial bundle.

By passage to limits over $V \subset R^{\infty}$, there results a classifying space $B(G ; E)$ for $E$-oriented stable $G$-bundles and a fibration sequence

$$
G \stackrel{e j}{\rightarrow} F E \stackrel{\tau}{\rightarrow} B(G ; E) \stackrel{q}{\rightarrow} B G .
$$

Now let $E$ be an $E_{\infty}$ ring spectrum, say a $\mathcal{S}$-spectrum. Then the results and definitions of $\$ \S 7$ and 11 combine to give that $B(G ; E)$ is a $\mathcal{G}$-space and $(*)$ is a sequence of $\mathcal{G}$-spaces and $\mathcal{G}$-maps. It follows that $B(G ; E)$ is an infinite loop space and, as such, is equivalent to the fibre of $B_{1}(e j): B G \simeq B_{1} G \rightarrow$ $B_{1} F E$. Thus $B_{1}(e j)$ is the universal obstruction to the $E$-orientability of stable $G$-bundles. There is also an integrally oriented version: $B(S G ; E)$ is equivalent to the fibre of $B_{1}(e j): B_{1} S G \rightarrow B_{1} S F E$.

We need one more bit of theory before we can turn to special cases. If $\psi$ : $E \rightarrow E$ is a map of ring spectra and $\mu$ is an $E$-orientation of a $G V$-bundle $\xi$, then $\psi \mu$ is another orientation of $\xi$ (where $\psi$ is thought of as a cohomology operation). Therefore $\psi \mu=\nu \mu$ for a unit $\nu \in E^{0}\left(X^{+}\right)$. On the classifying space level, there is a canonical map $c(\psi): B(G ; E) \rightarrow F E$ which corresponds to the functor which sends $(\xi, \mu)$ to $\nu$. The map $c(\psi)$ is called the universal "cannibalistic class" determined by $\psi$. (The justification-such as it is-for the name will appear in $\S 18$.) When $E$ is a $\mathcal{G}$-spectrum and $\psi$ is a map of $\mathcal{G}$-spectra, $c(\psi)$ is an infinite loop map (because it is the "quotient" $B \psi / 1$ of two $\mathcal{G}$-maps).

The most important example is $G=F$ and $E=k O$, where $k O$ is the $E_{\infty}$ ring spectrum obtained by application of the black box to the classifying space of the bipermutative category $\theta$ of real orthogonal groups. The zeroth space of $k O$ is equivalent to $B O \times Z$, and $S F k O \simeq B O \times\{1\}$ is usually written $B O_{\otimes}$. The map $B_{1} e: B S F \simeq B_{1} S F \rightarrow B_{1} B O_{\otimes}$ is the universal obstruction to the $k O$-orientability of integrally oriented stable spherical fibrations. By virtue of a general characterization of the connective ring spectrum 
associated to a periodic ring spectrum, $k O$ is equivalent as a ring spectrum to the spectrum obtained from the real Bott spectrum by killing its homotopy groups in negative degrees. The Adams operations $\psi^{r}: k O \rightarrow k O$ are maps of ring (but not of $E_{\infty}$ ring) spectra, where we have localized away from $r$, and bundle theoretical generalizations of the $d$ and $e$ invariants used by Adams [1] in his analysis of the groups $J S O(X)$ (the image of $[X, B S O]$ in $[X, B S F]$ ) can be defined in terms of the obstruction $B_{1} e$ and the universal cannibalistic classes $c\left(\psi^{r}\right)$.

Sullivan [74] characterized $B S$ Top as $B(S F ; k O)$, both localized away from 2, and here $B_{1} e: B S F \rightarrow B_{1} B O_{\otimes}$ is the universal obstruction to the reduction of a stable spherical fibration to a topological bundle. It is a standard consequence of surgery theory (Browder [17]) that a simply connected Poincaré duality space of dimension at least 5 is equivalent to a topological manifold if and only if its Spivak normal fibration $\xi$ has a reduction to a topological bundle. Away from 2, the composite of $B_{1} e$ and the classifying map $X \rightarrow B S F$ of $\xi$ is the only obstruction.

We are led to further questions. What does $B_{1} B O_{\otimes}$ really look like? Is $c\left(\psi^{r}\right): B(S F ; k O) \rightarrow B O_{\otimes}$ an infinite loop map? Are $B$ Top and $B(S F ; k O)$ equivalent away from 2 as infinite loop spaces or just as spaces?

Notes. See [48, II, IV, §3, and V] for details and related results. Some of this material is also joint work with Quinn and Ray. The classification theory on which it is based is given in [47]. Speculative discussions of the obstruction to $k O$-orientability were given by Adams [1, III, §7] and Sullivan [74]. The 2-primary obstruction to reducing a spherical fibration to a topological bundle has been analyzed in terms of transversality by Levitt and Morgan [35], Quinn [65, unpublished], Jones [29], and others and by homological study of the first delooping of $F /$ Top by Madsen and Milgram [41].

\section{ChaPter III. Descriptive ANALYSIS OF INFINITE LOOP SPACES}

15. General remarks on methodology. We have completed the development of the general machinery, and we are confronted with the problem of analyzing particular examples of its end products. For example, we want to decompose such infinite loop spaces as $F$, Top, and $B(S F ; k O)$ into products of simpler infinite loop spaces in so far as this is possible, one purpose being to reduce analysis of invariants of the infinite loop structure, such as homology operations, to their analysis on the relevant factors. In particular, we shall find that analysis of odd primary characteristic classes for topological bundles reduces to homological analysis of certain homomorphisms of finite groups.

We pause to discuss the techniques available. The theory of the preceding chapters is geared towards generic applications. All spaces with appropriate internal structure are infinite loop spaces, all maps which preserve this structure are infinite loop maps, all diagrams which commute up to structure-preserving homotopy commute on the infinite loop level. On the space level, the theory is wholly adequate: the machine succeeds in showing that all spaces which arise in the applications and which might reasonably be expected to be infinite loop spaces are in fact infinite loop spaces. On the map and diagram level, the machine is less successful. This is so because many of the maps which appear in the applications arise from particular 
representation theoretical or homotopical constructions rather than from generic constructions acceptable to the machine. In practice, this seems to remain true even with the generalized notion of "generic construction acceptable to the machine" given by $\S 6$.

The appropriate point of view is to cheerfully accept the limitations of the general machinery and to attack the new problems to which it gives rise armed with the old familiar tools of representation theory and homotopy theory. It should be noted that, with the possible exception of $\S 6$, the material of the previous chapters did not really lie in the domain of homotopy theory. It concerned very precise algebraic structures on spaces and spectra, the details of which become invisible upon passage to homotopy categories. The problems of infinite loop space theory look very different when seen through the eyes of homotopy theory, and some general discussion may clarify the later material.

Let $D$ and $E$ be (coordinatized) spectra. Restriction of maps of spectra $D \rightarrow E$ to $i$ th spaces yields an epimorphism $[D, E] \rightarrow \operatorname{proj} \lim \left[D_{i}, E_{i}\right]$, where the brackets denote spectra homotopy classes on the left and space homotopy classes on the right. In general, there is a kernel which is a proj $\lim ^{1}$ term. In the cases to be discussed here, the relevant proj $\lim ^{1}$ terms vanish either by finiteness arguments or by results of Anderson [5]. Thus $[D, E]$ and its relationship to $\left[D_{0}, E_{0}\right]$ can be studied by purely space level techniques.

It is advantageous to work one prime at a time, with all spaces and spectra localized or even completed at $p$. It is a pleasant fact that a map $D_{0} \rightarrow E_{0}$ is an infinite loop map if and only if its completion at each prime $p$ is an infinite loop map. It is even more pleasant that $[D, E] \rightarrow\left[D_{0}, E_{0}\right]$ is a monomorphism if and only if this is so after completion at each $p$. This monomorphism condition means that a map $D_{0} \rightarrow E_{0}$ can be infinitely delooped in at most one way. When it holds, a diagram of infinite loop maps which begins at $D_{0}$ and ends at $E_{0}$ will commute on the infinite loop level if it commutes on the space level.

Notes. See Sullivan [75] or Bousfield and Kan [15] for localizations and completions of spaces. These topics, for spaces and spectra, are also covered in [49], where the assertions of the last paragraph are proven (precise statements are in [48, II, §3]).

16. Transfer and results about $B S O$. We illustrate the general point of view by considering the transfer and its relationship with infinite loop maps. Let $(X, \theta)$ be a $\mathcal{C}$-space, where $\mathcal{C}$ is an $E_{\infty}$ operad, and let $\nu: E \rightarrow B$ be a principal $\pi$-bundle (or $p$-fold cover), where $\pi$ is the cyclic group of order $p$ with generator $\alpha$. Let $\mu: B \rightarrow B \pi \simeq \mathcal{C}(p) / \pi$ classify $\nu$, let $\tilde{\mu}: E \rightarrow \mathfrak{C}(p)$ cover $\mu$, and define $\bar{\nu}: B \rightarrow \mathcal{C}(p) \times{ }_{\pi} E^{p}$ by $\bar{\nu}(b)=\left(\tilde{\mu} e, \alpha e, \ldots, \alpha^{p} e\right)$ for any $e \in \nu^{-1}(b)$. For a homotopy class $g: E \rightarrow X$, the transfer of $g$, denoted $\tau(g)$, is the composite homotopy class

$$
B \stackrel{\tilde{\nu}}{\rightarrow} \mathcal{C}(p) \times_{\pi} E^{p^{1} \stackrel{1 \times g^{p}}{\rightarrow}} \mathcal{C}(p) \times_{\pi} X^{p} \stackrel{\theta_{p}}{\rightarrow} X
$$

If $\left(X^{\prime}, \theta^{\prime}\right)$ is another $\mathcal{C}$-space, an $H$-map $f: X \rightarrow X^{\prime}$ is said to commute with transfer if $\tau f_{*}(g)=f_{*}(\tau g)$. This is equivalent to homotopy commutativity of the diagram 


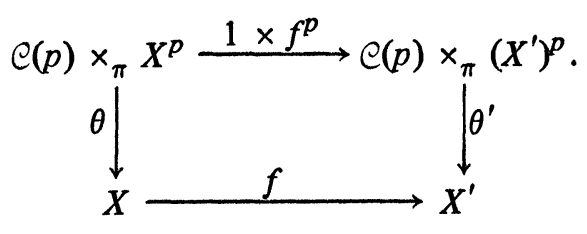

Obviously a $\mathcal{C}$-map, or an infinite loop map, commutes with transfer. Generically, it is clear that a transfer commuting map between infinite loop spaces cannot reasonably be expected to be an infinite loop map since the transfer sees only a fraction of the entire $E_{\infty}$ structure.

Henceforward, complete all spaces at $p$ and consider the case of $B S O$. Here we have complete information on $[B S O, B S O]$. An $H$-map $f: B S O \rightarrow B S O$ is essentially just a linear combination of Adams operations $\psi^{r}$. A representation theoretical calculation due to Madsen, Snaith, and Tornehave [42] demonstrates that $f$ commutes with transfer only if $f$ is a linear combination of the $\psi^{r}$ with $r$ prime to $p$. But such maps $f$ are infinite loop maps by Bott periodicity. Thus, in this special case, an $H$-map which commutes with transfer is necessarily an infinite loop map.

This criterion for a map $B S O \rightarrow B S O$ to be an infinite loop map is attractive, but not useful as it stands. The maps one really wants to study involve spaces of the same homotopy type as $B S O$ but with a (seemingly) different infinite loop space structure, such as $B S O_{\otimes}$ and, if $p>2, F /$ Top. Adams and Priddy [3] have proven that any such $X$ is in fact equivalent to $B S O$ as an infinite loop space. We outline their proof. Let bso be the 1-connected cover of $k O$, with zeroth space $B S O$, and let $X \simeq B S O$ be the zeroth space of a 1-connected spectrum $E$. By examination of the spectral sequence for computing the $\bmod p$ cohomology of $E$ derived from its Postnikov tower, one finds that $H^{*} E$ is isomorphic to $H^{*} b s o$, a known module over the Steenrod algebra $A$. The isomorphism may be thought of as an element of $\operatorname{Ext}_{\dot{A}}^{0,0}\left(H^{*} b s o, H^{*} E\right)$, and a good deal of purely homological calculation demonstrates that $\operatorname{Ext}_{A}^{s, t}\left(H^{*} b s o, H^{*} E\right)=0$ if $s>0$ and $t-s=$ -1 . Thus the isomorphism is a permanent cycle in the relevant Adams spectral sequence. While that spectral sequence presumably need not coverage to $[E, b s o], E$ is the completion at $p$ of a $C W$-spectrum with finite skeleta, and restriction to these skeleta leads to construction of the required equivalence $E \rightarrow$ bso.

Now given $X$ and $Y$, two infinite loop spaces with underlying spaces of the homotopy type of $B S O$, we are entitled to conclude that any transfer commuting $H$-map $X \rightarrow Y$ is an infinite loop map.

We note another consequence. For $p$ odd, where $B O \simeq B S O$, we conclude that $B_{1} B O_{\otimes}$ is equivalent to $(b s o)_{1} \simeq S U / S O$, the Bott delooping of $B O$. Thus the $p$-primary obstruction to the reduction of a spherical fibration over $X$ to a topological bundle is an element of $[X, S U / S O]$, and the reservoir of existing information and techniques in $K$-theory can be brought to bear on the analysis of the obstruction.

Notes. See Kahn and Priddy [32] for details and greater generality on transfer. The papers of Adams and Priddy [3] and Madsen, Snaith, and Tornehave [42] contain many further related results (some of which will be discussed in the following sections). 
17. The uniqueness of deloopings. Again, all spaces and spectra are to be completed at $p$. To chase diagrams on the infinite loop level, we need to know the unique deloopability of maps between certain infinite loop spaces.

Madsen, Snaith, and Tornehave [42] proved this for maps $B S O \rightarrow B S O$ and, if $p>2, S O \rightarrow B S O$. The latter result asserts that, if $p>2$, there are no nontrivial maps of spectra $s o \rightarrow b s o$ (where $s o=\Omega b s o$ ). Ligaard [36] later proved that there are no nontrivial maps $s o \rightarrow b s o$ and spin $\rightarrow b$ so when $p=2$ (where spin is the 1-connected cover of so). The proofs go by inductive analysis of Postnikov towers and are based on results of Anderson and Hodgkin [6] which give $K O^{*}(K(\pi, n))=0$ if $\pi$ is finite and $n \geqslant 2$ or if $\pi=\hat{Z}_{p}$ and $n \geqslant 3$.

To proceed further, we must introduce additional spaces. With $B O, B O_{\otimes}$, and cognate spaces, these will be the basic building blocks out of which many of the classifying spaces of geometric topology are built. Let $r=3$ if $p=2$ and let $r$ reduce mod $p^{2}$ to a generator of the group of units of $Z_{p^{2}}$ if $p>2$; for later use, pick $r$ to be a power of an odd prime $q$. Define $J$ and $J_{\otimes}$ to be the fibres of $\psi^{r}-1: B O \rightarrow B$ Spin and $\psi^{r} / 1: B O_{\otimes} \rightarrow B \operatorname{Spin}_{\otimes}$ and define $B C$ to be the fibre of $c\left(\psi^{r}\right): B(S F ; k O) \rightarrow B \operatorname{Spin}_{\otimes}$. Here $B \operatorname{Spin}_{\otimes}$ is the 2-connected cover of $B O_{\otimes}$ as an infinite loop space (and the required lifts to $B$ Spin and $B \operatorname{Spin}_{\otimes}$ exist and are unique). Define $C=\Omega B C . J$ is short for "Image $J$ " and $C$ is short for "Cokernel $J$ ", the names coming from the relationship between the homotopy groups of these spaces and the classical " $J$-homomorphism" $\pi_{*} S O \rightarrow \pi_{*} S F$.

Clearly $\psi^{r}-1$ is an infinite loop map and $J$ is an infinite loop space. It is an easy consequence of the Adams-Priddy result on the unique deloopability of $B S O$ that $\psi^{r} / 1$ is also an infinite loop map and thus that $J_{\otimes}$ is an infinite loop space. We shall later see that $c\left(\psi^{r}\right)$ is an infinite loop map and thus that $B C$ and $C$ are infinite loop spaces.

For the diagrams we wish to chase, the crucial fact is the unique deloopability of maps $S F \rightarrow B O_{\otimes}$ or, equivalently since $B O_{\otimes} \simeq R P P^{\infty} \times B S O_{\otimes}$ and $B S O \simeq B S O_{\otimes}$ as infinite loop spaces, $S F \rightarrow B S O$. Madsen, Snaith, and Tornehave [42] and Ligaard [36] used their results cited above and the fibration defining $J_{\otimes}$ to show the unique deloopability of maps $J_{\otimes} \rightarrow B S O$. This implies the conclusion for $S F$ via the results of the following two paragraphs.

We have a composite infinite loop map $\iota: C \rightarrow S F$ (namely $C \rightarrow \Omega B(S F$; $k O) \rightarrow \Omega B S F \simeq S F$ ), and the Adams conjecture (Quillen [61], Sullivan [75]) gives a map $\alpha: J \rightarrow S F$; $\alpha$ is not even a map of $H$-spaces when $p=2$ [39], [20, II, \$12], but it is conjectured that $\alpha$ is an infinite loop map when $p>2$. The composite of $\alpha \times \imath$ and the product $S F \times S F \rightarrow S F$ is an equivalence $J \times C$ $\rightarrow S F$, this being an interpretation due to Sullivan of work of Adams [1]. More precisely, there is an infinite loop map $e: S F \rightarrow J_{\otimes}$, to be discussed in $\S 19$, which has fibre $C$ and whose composite with $\alpha$ is an equivalence $J \rightarrow J_{\otimes}$. On homotopy groups, $e$ induces a version of Adams' $e$-invariant. (See $[48, \mathrm{~V}$, §3-5] for details.)

It is a basic result of Hodgkin and Snaith [27], [70] that there are no nontrivial maps $C \rightarrow B S O$ on either the space or the spectrum level. We sketch a proof. It is an immediate consequence of the fact that $Q S^{0}$ is a group completion of $\amalg B \Sigma_{j}$ that there is a map from $B \Sigma_{\infty}$ to the zero component 
$Q_{0} S^{0}$ of $Q S^{0}$ which induces an isomorphism on integral homology and therefore on cohomology with arbitrary coefficients. This reduces the calculation of $K^{*}\left(Q_{0} S^{0}\right)$ to representation theory; see Hodgkin [26]. It turns out that the unit $e: Q S^{0} \rightarrow B O \times Z$ of $k O$ induces an epimorphism $K^{*}(B O) \rightarrow$ $K^{*}\left(Q_{0} S^{0}\right)$. By translation to 1-components, this also holds for $e: S F \rightarrow B O_{\otimes}$. However, this map $e$ factors through $e: S F \rightarrow J_{\otimes}$, which must therefore also induce an epimorphism on $K^{*}$. By the splitting of $S F$, this implies $K^{*}(C)=0$. Standard arguments then give that $K O^{*}(C)=0$ on both the space and the spectrum level.

18. The $k O$ orientations of Spin and $S$ Top bundles. We return to the context of $\$ 14$ armed with the results of the previous sections.

Atiyah, Bott, and Shapiro [9] constructed a canonical $k O$-orientation of Spin bundles, and there results an $H$-map $g: B$ Spin $\rightarrow B(S F ; k O)$. (There also results a map $M$ Spin $\rightarrow k O$ of ring spectra, via the orientations of the universal bundles, but this point of view will not concern us here.) The composite of $g$ and $c\left(\psi^{r}\right): B(S F ; k O) \rightarrow B \operatorname{Spin}_{\otimes}$ (away from $r$ ) is called $\rho^{r}$. By Adams [1, II], it is the cover of a map $\rho^{r}: B S O \rightarrow B S O_{\otimes}$. One thinks of $\rho^{r}$ as resulting from $k O$ feeding on its own cohomology operation $\psi^{r}$, hence the term cannibalistic class for such operations. Madsen, Snaith, and Tornehave [42] noted that $\rho^{r}$ is transfer commuting (at any $p$ prime to $r$ ) and is therefore an infinite loop map.

The space $S F /$ Spin classifies Spin bundles with a trivialization of their underlying spherical fibrations. There is a canonical map $f: S F /$ Spin $\rightarrow B O_{\otimes}$ which corresponds on the bundle level to the unit by which the Atiyah-BottShapiro orientation differs from the pullback along the trivialization of the canonical orientation of the trivial bundle. The following diagram is homotopy commutative:

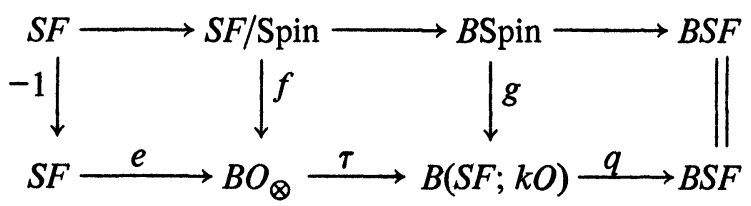

By diagram chases from the results of the previous sections [42] or [48, V, §7], one can deduce that $f$ is an infinite loop map at each prime $p$ and therefore globally. By use of the uniqueness of deloopings of maps $S F \rightarrow B O_{\otimes}$, it follows easily that the diagram is commutative on the infinite loop space level.

At $p=2$, the following composite is an equivalence of infinite loop spaces:

$$
B C \times B \operatorname{Spin} \stackrel{i \times g}{\rightarrow} B(S F ; k O) \times B(S F ; k O) \stackrel{\phi}{\rightarrow} B(S F ; k O),
$$

where $i$ is the natural map and $\phi$ is the product. At $p>2, B O$, or any equivalent infinite loop space, splits as the product of two infinite loop spaces $W$ and $W^{\perp}$, where $W$ carries the homotopy groups in degrees $2 i(p-1)$ and $W^{\perp}$ carries the remaining homotopy groups (Adams [2], Peterson [59]). Here the following composite is an equivalence of infinite loop spaces:

$$
B C \times W \times W^{\perp} \rightarrow B C \times B O \times B O_{\otimes} \stackrel{i \times g \times \tau}{\rightarrow} B(S F ; k O)^{3} \stackrel{\phi}{\rightarrow} B(S F ; k O) .
$$


On the space level, this is essentially an interpretation due to Sullivan [74] of results of Adams [1]; details are in [48, V].

Sullivan [74] constructed a canonical $k O\left[\frac{1}{2}\right]$-orientation of $S$ Top bundles. Localizing all spaces away from 2 , there results a map $\bar{g}$ : $B S$ Top $\rightarrow$ $B(S F ; k O)$. The following composite (away from 2 and $r$ ) is called $\theta^{r}$ :

$$
B S O \rightarrow B S \text { Top } \stackrel{\bar{g}}{\rightarrow} B(S F ; k O) \stackrel{c\left(\psi^{r}\right)}{\rightarrow} B S O_{\otimes} .
$$

By comparison of $\rho^{r}$ and $\theta^{r}$ on 2-plane bundles, Sullivan [74] showed that $\theta^{r} \simeq \rho^{r}\left(2 \psi^{2}+\psi^{r}\right)$. Therefore $\theta^{r}$ is an infinite loop map.

Again, the unit by which the two obvious $k O\left[\frac{1}{2}\right]$-orientations of a $S$ Top bundle with a trivialization as a spherical fibration differ corresponds to a map $\bar{f}: F /$ Top $\rightarrow B O_{\otimes}$ (away from 2). Sullivan [73], [74] proved that $\bar{f}$ is an equivalence. The following diagram is homotopy commutative, and therefore $\bar{g}$ is also an equivalence:

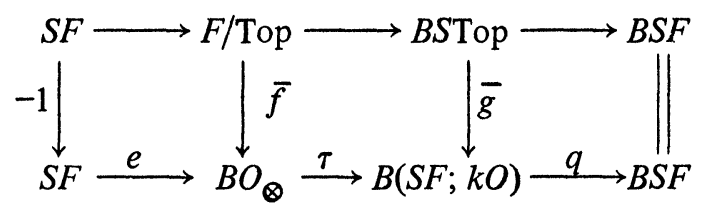

By diagram chases from the results of the previous sections [42] or [48, $\mathrm{V}, \S 7$, one can deduce that $\bar{f}$ is an infinite loop map at each prime $p>2$ and therefore globally (i.e., away from 2). By the uniqueness of delooping of maps $S F \rightarrow B O_{\otimes}$, it follows easily that the diagram is commutative on the infinite loop space level. In particular, $\bar{g}$ is an equivalence of infinite loop spaces.

Thus to study invariants of the infinite loop space $B S$ Top away from 2 it suffices to study $B(S F ; k O)$, and, at each odd prime $p$, this study in turn reduces to the study of $B C \times W \times W^{\perp}$ and thus of $B C$ (since $W$ and $W^{\perp}$ are well understood). We must still find out why $B C$ is an infinite loop space and how to study its invariants.

Notes. There does not yet exist a descriptive analysis of $B$ Top as an infinite loop space at $p=2$. Madsen and Milgram [41] have shown that $B_{2}(F /$ Top $)$ at $p=2$ is the appropriate product of Eilenberg-Mac Lane spaces, whereas Madsen [38] showed that $B_{3}(F /$ Top) does not so split.

19. Brauer lift, Frobenius, and finite fields. Quillen [61], [62], in his proof of the Adams conjecture and his computation of the algebraic $K$-groups of finite fields, established certain basic equivalences between spaces associated to finite fields and their algebraic closures and the classifying spaces of topological $K$-theory. Generalizations of his results to the infinite loop space level (and from the general linear to the orthogonal groups of finite fields) lead to the desired infinite loop space structure on $B C$ and to reduction of the analysis of its homology to computations of the homology of appropriate finite groups. It also leads to a splitting $S F=J \times C$ of infinite loop spaces at each odd prime and to an infinite loop fibration $C \rightarrow S F \rightarrow J_{\otimes}$ at $p=2$.

Fix a prime $p$ and complete all spaces at $p$. Let $r=q^{a}$ for an odd prime $q \neq p$ be as in $\S 17(r=3$ if $p=2)$. Let $k_{r}$ be a field with $r$ elements and let $\bar{k}_{q}$ be an algebraic closure of the field with $q$ elements. Define $k O^{\delta}=\mathbf{B}\left(B \mathbb{K}_{q}\right)$, the completion at $p$ of the $E_{\infty}$ ring spectrum produced by application of the black box to the classifying space of the bipermutative category of orthogonal 
groups of $\bar{k}_{q}$. Here and below, the superscript $\delta$ is to be read "discrete model for".

By use of Brauer lifting of appropriate representations, one can construct a map $\lambda$ of ring spaces from the zeroth space of $k O^{\delta}$ to the zeroth space of $k O$. Quillen's calculations [61] imply that $\lambda$ induces an isomorphism on mod $p$ cohomology and is therefore an equivalence (because we have completed and not just localized at $p$ ). By virtue of a general characterization of the connective ring spectrum associated to the periodic ring spectrum determined by a periodic ring space, it follows immediately that $\lambda$ extends uniquely to an equivalence of ring spectra $\lambda: k O^{\delta} \rightarrow k O$. As was first proven by Tornehave [77], there is an analogous equivalence $k U^{\delta} \rightarrow k U$, where $k U^{\delta}=\mathbf{B}\left(B \mathcal{G} E \bar{k}_{q}\right)$.

Let $\phi^{r}: \theta \bar{k}_{q} \rightarrow \theta \bar{k}_{q}$ be the Frobenius automorphism (which maps $x$ to $x^{r}$ on matrix entries). Then the following diagram of spectra homotopy commutes by the general characterization cited above and the fact that $\lambda \phi^{r} \approx \psi^{r} \lambda$ on zeroth spaces by an easy representation theoretical calculation:

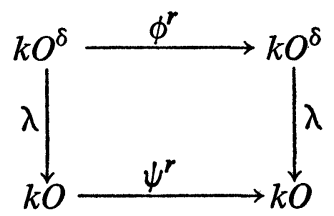

By the transfer criterion of $\S 16$ and a representation theoretical calculation originally due to Tornehave [unpublished], the equivalence $\lambda: B O_{\otimes}^{\delta} \rightarrow B O_{\otimes}$ obtained by restriction of $\lambda$ to 1-components of zeroth spaces is an infinite loop map. By use of the uniqueness of deloopings of maps $S F \rightarrow B O_{\otimes}$, the following is easily seen to be a commutative diagram of infinite loop spaces, where the rows are orientation sequences as constructed in $\S 14$.

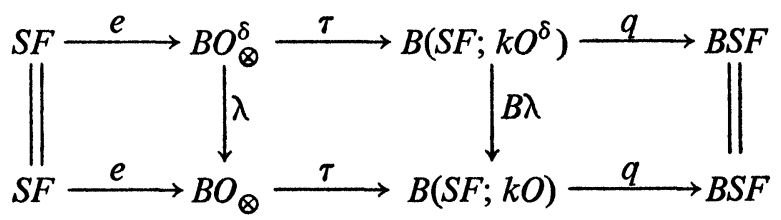

Therefore $B \lambda$ is an equivalence of infinite loop spaces.

Define $B C^{\delta}$ to be the fibre of $c\left(\phi^{r}\right): B\left(S F ; k O^{\delta}\right) \rightarrow B \operatorname{Spin}_{\otimes}^{\delta}$ (where $B \operatorname{Spin}_{\otimes}^{\delta}$ is the 2-connected cover of $\left.B O_{\otimes}^{\delta}\right)$. There results an equivalence of fibration sequences

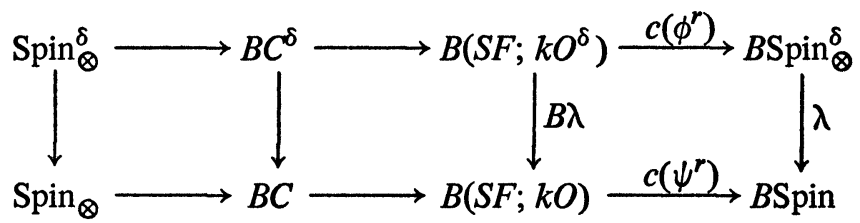

The functor $\phi^{r}: \theta \bar{k}_{q} \rightarrow \vartheta \bar{k}_{q}$ preserves $\oplus$ and $\otimes$ and is in fact a morphism of bipermutative categories. Therefore its induced map $\mathbf{B}\left(B \bullet \bar{k}_{q}\right) \rightarrow \mathbf{B}\left(B \cup \bar{k}_{q}\right)$ is a map of $E_{\infty}$ ring spectra. It follows that $c\left(\phi^{r}\right)$ is an infinite loop map. Thus, by the diagram above, $c\left(\psi^{r}\right)$ is an infinite loop map and $B C \simeq B C^{\delta}$ is an infinite loop space. 
Let $j$ be the fibre of $\psi^{r}-1: k O \rightarrow b$ spin (where $b$ spin is the 2-connected cover of $k O$ ). Then $J$ and $J_{\otimes}$ are the 0 -component and 1-component of the zeroth space of $j$. When $p>2$, define $j^{\delta}=\mathbf{B}\left(\Theta k_{r}\right)\left(\mathcal{G} k_{r}\right.$ would work equally well). When $p=2$, let $\mathscr{T} k_{3}$ be the subbipermutative category of $\Theta k_{3}$ whose morphisms $N\left(n, k_{3}\right): n \rightarrow n$ are those matrices with equal spinor norm and determinant and define $j^{\delta}=\mathbf{B}\left(B \Im k_{3}\right)$. Let $J^{\delta}$ and $J_{\otimes}^{\delta}$ denote the 0-component and 1-component of the zeroth space of $j^{\delta}$.

Clearly $j$ is equivalent to the fibre of $\phi^{r}-1: k O^{\delta} \rightarrow b \operatorname{spin}^{\delta}$ (where $b \operatorname{spin}^{\delta}$ is the 2-connected cover of $k O^{\delta}$ ). The composite of $\phi^{r}-1$ and the natural map $j^{\delta} \rightarrow k O^{\delta}$ is trivial (since elements of $O\left(n, k_{r}\right)$ are left fixed by $\phi^{r}$ ), and there results a lift $\nu: j^{\delta} \rightarrow j$. By use of calculations of Quillen [62] when $p>2$ and of Fiedorowicz and Priddy [24] when $p=2$, one can verify that $\nu$ is an equivalence. Observe the effect of this: $j$ was just a spectrum, with no additional structure, but is now seen to be equivalent to an $E_{\infty}$ ring spectrum. On zeroth spaces, $\nu$ restricts to equivalences $J^{\delta} \rightarrow J$ and $J_{\otimes}^{\delta} \rightarrow J_{\otimes}$ of additive and multiplicative infinite loop spaces.

The composite of the natural map $B\left(S F ; j^{\delta}\right) \rightarrow B\left(S F ; k O^{\delta}\right)$ and $c\left(\phi^{r}\right)$ : $B\left(S F ; k O^{\delta}\right) \rightarrow B \operatorname{Spin}_{\otimes}^{\delta}$ is the trivial infinite loop map (again because elements of $O\left(n, k_{r}\right)$ are fixed by $\left.\phi^{r}\right)$, and there results an infinite loop map $B\left(S F ; j^{\delta}\right) \rightarrow B C^{\delta}$ which turns out to be an equivalence. Thus $B C$ may be regarded as the classifying space for $j^{\delta}$-oriented spherical fibrations. The orientation sequence

$$
S F \stackrel{e}{\rightarrow} J_{\otimes}^{\delta} \stackrel{\tau}{\rightarrow} B\left(S F ; j^{\delta}\right) \stackrel{q}{\rightarrow} B S F
$$

is the basic tool for the homological analysis of $B C$. Under the equivalence $J_{\otimes} \simeq J_{\otimes}^{\delta}, e$ is the map discussed in $\S 17$.

Recall from $\S \S 10$ and 12 that $S F$ can be regarded as the 1-component of the zeroth space of $\mathbf{B}(B \mathcal{E})$, where $\mathcal{E}$ is the category of finite sets, and that $e$ is derived from a morphism $\mathcal{E} \rightarrow\left(\vartheta k_{r}\right.$ (if $p>2$ ) or $\mathcal{E} \rightarrow \mathfrak{N} k_{3}$ (if $p=2$ ) of bipermutative categories. Thus, by the group completion property of the recognition principle, analysis of $e_{*}: H_{*} S F \rightarrow H_{*} J_{\otimes}^{\delta}$ reduces to homological analysis of the homomorphismis $\Sigma_{n} \rightarrow O\left(n, k_{r}\right)$ or $\Sigma_{n} \rightarrow N\left(n, k_{3}\right)$. In view of the results of the previous section, this analysis will also determine the characteristic classes for stable topological bundles when $p>2$.

By regarding elements of $O\left(n, k_{r}\right)$ as permutations of the set $\left(k_{r}\right)^{n}$ of $r^{n}$ elements, one obtains a morphism $\left(\vartheta k_{r}, \oplus\right) \rightarrow(\mathcal{E}, \otimes)$ or $\left(\Re k_{3}, \oplus\right) \rightarrow(\mathcal{E}, \otimes)$ of permutative categories. By use of the recognition principle (in particular, the last paragraph of $\S 12$ with $\left.M=\left\{r^{n}\right\}\right)$, there results a commutative diagram of infinite loop spaces

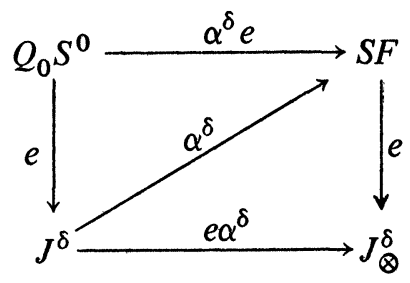

(both maps $e$ being restrictions of the unit map $e: Q_{\infty} S^{0} \rightarrow j^{\delta}$ ). Tornehave 
[79] showed by a representation theoretical argument, and I later showed by direct homological calculation, that $e \alpha^{\delta}$ is an equivalence when $p>2$. It follows that the composite of $\alpha^{\delta} \times \iota: J^{\delta} \times C \rightarrow S F \times S F$ and the product $S F \times S F \rightarrow S F$ is an equivalence of infinite loop spaces. It is conjectured that $\alpha^{\delta}$ agrees under the equivalence $J \simeq J^{\delta}$ with a map $\alpha$ which arises from a solution to the Adams conjecture.

Notes. See [48, VIII] for proofs and related results. The cited chapter is written jointly with Jørgen Tornehave and contains the material of his unpublished works [77], [78], [79].

\section{Chapter IV. HoMOlogiCAL ANALYSIS OF INFINITE LOOP SPACES}

20. Homology operations on $E_{\infty}$ spaces. Except where otherwise stated, all homology in this chapter is to be taken with $\bmod p$ coefficients for a fixed prime $p$.

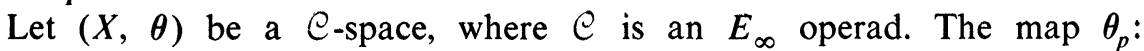
$\mathcal{C}(p) \times_{\Sigma_{p}} X^{p} \rightarrow X$ gives rise to natural homomorphisms (homology analogs of Steenrod operations)

$$
Q^{r}: H_{q} X \rightarrow H_{q+r} X \quad \text { if } p=2 \text { and } Q^{r}: H_{q} X \rightarrow H_{q+2 r(p-1)} X \text { if } p>2 \text {. }
$$

These operations vanish if $p=2$ and $r<q$ or if $p>2$ and $2 r<q$; they give the $p$ th power if $p=2$ and $r=q$ or if $p>2$ and $2 r=q$. For $r>0, Q^{r}$ annihilates the identity element of $H_{*} X$. There is an external Cartan formula of the form

$$
Q^{r}(x \otimes y)=\sum_{i+j=r} Q^{i} x \otimes Q^{j} y,
$$

and both the product and the coproduct on $H_{*} X$ commute with the operations. There are Adem relations for the iterated operations which read as follows when $p=2$ :

$$
Q^{r} Q^{s}=\sum_{i}(2 i-r, r-s-i-1) Q^{r+s-i} Q^{i}
$$

The operations commute with the homology suspension $\sigma_{*}: \tilde{H}_{*} \Omega X \rightarrow H_{*} X$,

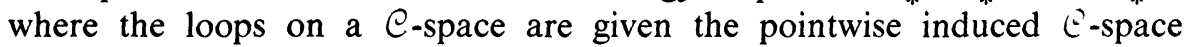
structure.

Of course, these properties are reminiscent of the standard properties of the Steenrod operations $P^{r}\left(P^{r}=\mathrm{Sq}^{r}\right.$ if $\left.p=2\right)$ in the cohomology of spaces [72]. There are differences. The $P^{r}$ begin with $P^{0}=1$ and end with the $p$ th power, whereas the $Q^{r}$ begin with the $p$ th power. The coefficient in the Adem relations seems somehow reversed in the two situations.

A brief description of how the operations arise may clarify the comparison. Let $V$ be a $Z_{p}\left(\Sigma_{p}\right)$-free resolution of $Z_{p}$ and let $W$ be the standard $Z_{p}(\pi)$-free resolution of $Z_{p}$ with one basis element $e_{i}$ in each degree $i \geqslant 0$, where $\pi$ is cyclic of order $p$. Embed $W$ in $V \pi$-equivariantly. Let $K$ be a ( $Z$ graded) chain complex over $Z_{p}$ and let $K^{p}$ denote the $p$-fold tensor product of $K$ with itself. Then the homology $H\left(V \otimes_{\Sigma_{p}} K^{p}\right)$ is generated by elements of the form $e_{0} \otimes x_{1} \otimes \cdots \otimes x_{p}$ for $x_{j} \in H K^{p}$ and $e_{i} \otimes x^{p}$ for $x \in H_{q} K$, where only those $i$ of the form $(2 r-q)(p-1)-\varepsilon$ with $\varepsilon=0$ or 1 appear when $p>2$. Given a morphism $\theta_{p}: V \otimes_{\Sigma_{p}} K^{p} \rightarrow K$ of chain complexes, the elements 
$e_{i} \otimes x^{p}$ give rise to operations upon passage to homology.

In our $\mathcal{C}$-space situation, the map $\theta_{p}$ induces a chain map $\theta_{p}: C_{*} \mathcal{E}(p)$ $\otimes_{\Sigma_{p}}\left(C_{*} X\right)^{p} \rightarrow C_{*} X$ by use of the standard shuffle map $C_{*} X \otimes C_{*} Y \rightarrow$ $C_{*}(X \times Y)$, and $C_{*} \mathcal{e}(p)$ is a $Z_{p}\left(\Sigma_{p}\right)$-free resolution of $Z_{p}$ since $\mathcal{C}$ is an $E_{\infty}$ operad. In the cohomology situation, an acyclic models argument gives a natural chain map $V \otimes_{\Sigma_{p}}\left(C^{*} Y\right)^{p} \rightarrow C^{*} Y$ for spaces $Y$ except that, to make sense of this, one must regrade the cochains $C^{*} Y$ by nonpositive subscripts (or regrade $V$ by nonpositive superscripts). In both situations, when $p>2$, the $i$ of the form $(2 r-q)(p-1)$ give rise to the operations and the $i$ of the form $(2 r-q)(p-1)-1$ give rise to their Bocksteins.

The difference in grading fully accounts for the differences between Steenrod operations and homology operations. Their similarities are accounted for as well: the chains on $E_{\infty}$ spaces and the cochains on spaces both give functors to the algebraic category which is the appropriate domain of definition of (generalized) Steenrod operations. The cobar construction on cocommutative Hopf algebras gives another such functor of considerable interest. Under appropriate algebraic hypotheses, we verify such things as the Cartan formula, the Adem relations, and commutation with suspension once and for all in the general algebraic context, We then deduce such results for the particular case at hand by checking that the appropriate hypotheses are satisfied. For example, the Adem relations for the homology operations come from the following diagram, the commutativity of which is part of the definition of an action by an operad:

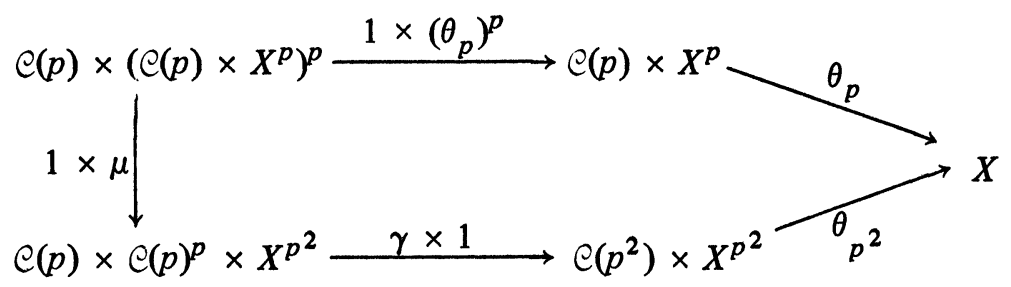

where $\mu$ is the evident shuffle homeomorphism.

The homology operations $Q^{r}$ are related to the duals $P_{*}^{r}$ of the Steenrod operations by the Nishida relations [57], which are deduced by naturality from computation of the $P_{*}^{r}$ on $H_{*}\left(C(p) \times_{\Sigma_{p}} X^{p}\right)$. When $p=2$, they read

$$
P_{*}^{r} Q^{s}=\sum_{i}(r-2 i, s-2 r+2 i) Q^{s-r+i} P_{*}^{i} .
$$

When $X$ is an infinite loop space, the homology operations commute with $\chi$, the conjugation in the Hopf algebra $H_{*} X$ derived from the inverse map.

There are also analogs 2: $H_{q}\left(X ; Z_{p^{i}}\right) \rightarrow H_{p q}\left(X ; Z_{p^{i+1}}\right)$ of the Pontryagin $p$ th powers in cohomology (see Thomas [76]).

Notes. See [43] and [20, I, §§1, 7] for details. The mod 2 homology operations were first introduced by Araki and Kudo [7], and a simpler treatment was later given by Browder [16]. The $\bmod p$ operations for $p>2$ were first introduced by Dyer and Lashof [22]. When $p=2$, the operations :were introduced by Madsen [40]. 
21. The Dyer-Lashof algebra; $H_{*} C X$ and $H_{*} Q X$. Pursuing the analogy with Steenrod operations in cohomology, we define the Dyer-Lashof algebra $R$ to be the quotient of the free commutative algebra generated by the operations $Q^{r}$ and, if $p>2, \beta Q^{r+1}$ for $r \geqslant 0$ by the ideal generated by those elements which annihilate every homology class of every $E_{\infty}$ space. $R$ is a Hopf algebra and has a basis consisting of iterated operations $Q^{I}=$ $\beta^{\varepsilon_{1}} Q^{s_{1}} \ldots \beta^{\varepsilon_{k}} Q^{s_{k}}$ for certain admissible sequences $I=\left(\varepsilon_{1}, s_{1}, \ldots, \varepsilon_{k}, s_{k}\right)$, the $\beta$ 's and $\varepsilon$ 's being absent when $p=2$. Such monomials of length $k$ span a subcoalgebra of $R$; its dual is a polynomial algebra on $k$ generators when $p=2$ but has a considerably more complicated structure when $p>2$, the complications arising from the Bockstein operations.

We define an allowable $R$-structure (module, Hopf algebra, Hopf algebra with conjugation, etc.) to be an $R$-structure which satisfies the constraints dictated by the properties of the operations discussed in the previous section (in particular, the vanishing of the operations below the $p$ th power). We then construct free allowable $R$-structures. In particular, we have the free allowable $R$-Hopf algebra $U H$ and the free allowable $R$-Hopf algebra with conjugation $G U H$ generated by a (suitable) coalgebra $H$. Noninvariantly, $U H$ can be described as the free commutative algebra generated by elements of the form $Q^{I} x$ for appropriate admissible monomials $I$ (depending on the degree of $x$ ), where $x$ runs through a basis for $H$ (with unit element deleted since the $Q^{r}$ for $r>0$ are to annihilate the identity element). The coproduct on $H$ extends to $U H$ by the Cartan formula and commutation with the product. The $R$ operations are determined by the Adem relations. If $H$ has Steenrod operations, then these extend to $U H$ by the Nishida relations and the Cartan formula. GUH is the localization of the algebra $U H$ at its (appropriately defined) multiplicative submonoid of components. The coproduct, $R$ operations, and, if present, Steenrod operations extend from $U H$ to $G U H$ by commutation with the conjugation.

Let $C$ be the monad associated to an $E_{\infty}$ operad $\Theta$. By freeness, the natural maps $\eta: X \rightarrow C X$ and $\eta: X \rightarrow Q X$ induce morphisms

$$
\bar{\eta}: U H_{*} X \rightarrow H_{*} C X \text { and } \tilde{\eta}: G U H_{*} X \rightarrow H_{*} Q X
$$

which preserve all structure in sight. The essential starting point of the applications of homology operations is the fact that $\bar{\eta}$ and $\tilde{\eta}$ are isomorphisms. For $Q X$, modulo care needed when $X$ is not connected, the proof proceeds by application of the comparison theorem to the Serre spectral sequence of the fibrations $Q X \rightarrow P Q \Sigma X \rightarrow Q \Sigma X$ and is quite analogous to Serre's procedure [68] (see [43] when $p>2$ ) for the calculation of the cohomology of $K(\pi, n)$ 's. For $C X$, we first note that the commutative

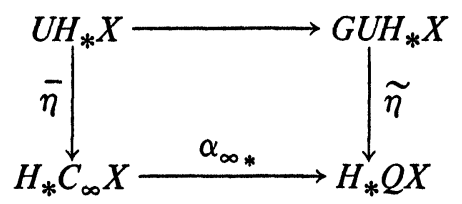

shows that $\bar{\eta}$ is a monomorphism when $\mathcal{E}=\mathfrak{e}_{\infty}$ and thus for any $\mathcal{E}$ (by the equivalences $\left.C X \leftarrow\left(C \times C_{\infty}\right)(X) \rightarrow C_{\infty} X\right)$. That $\bar{\eta}$ is an epimorphism is then 
proven by induction over the filtration $\left\{F_{k} C X\right\}$ by exploitation of the structural maps $\gamma$ of the operad $C$.

These calculations imply the case $n=\infty$ of the approximation theorem discussed in $\$ 4$.

Since $C S^{0}=\amalg K\left(\Sigma_{j}, 1\right)$, the calculation just sketched includes a rederivation of Nakaoka's fundamental results [55], [56] on the homology of symmetric groups. The operad structure incorporates the iterated wreath products essential to the algebraic understanding of these homology groups.

The calculations discussed here have played a vital role in the proofs of three very beautiful theorems in homotopy theory. Let $Q_{0} S^{0}$ be the component of the trivial loop in $Q S^{0}$. Kahn and Priddy [31], [32] used these calculations and the transfer to prove that if $f: R P^{\infty} \rightarrow Q_{0} S^{0}$ is any map nontrivial on $\pi_{1}$, then the induced map $Q R P^{\infty} \rightarrow Q_{0} S^{0}$ induces an epimorphism on homotopy groups; they also proved an analogous result at odd primes. Building on the Kahn-Priddy theorem, Nishida [58] proved the long conjectured assertion that every element of $\pi_{n}^{s}, n>0$, is nilpotent. Finally, along different lines, Curtis [21] proved that the Hopf maps and (if present) the Arf invariant classes in dimensions $2^{i}-2$ are the only elements of the 2-primary component of $\pi_{*}^{s}$ which can have nontrivial image under the $(\bmod 2)$ Hurewicz homomorphism of $Q S^{0} .^{3}$

Notes. See [20, I, §§2-5] for details. The calculation of $H_{*} Q X$ is due to Dyer and Lashof [22], explicitly for connected $X$ and implicitly otherwise. The calculation of $H_{*} C X$ was implicit in the work of Nakaoka [55], [56] and was long part of the folklore. When $p=2$, the dual of $R$ was first analyzed by Madsen [38], [39].

22. The homology of $E_{\infty}$ ring spaces; $H_{*} C\left(X^{+}\right), H_{*} Q\left(X^{+}\right), H_{*} S F$. Let $(X, \theta, \xi)$ be a $(\mathcal{C}, \mathcal{G})$-space, where $\mathcal{C}$ and $\mathcal{G}$ are $E_{\infty}$ operads. Then $H_{*} X$ has a Pontryagin product $*$ and homology operations $Q^{r}$ coming from the additive $E_{\infty}$ structure $(X, \theta)$ and a Pontryagin product \# and homology operations $\tilde{Q}^{r}$ coming from the multiplicative $E_{\infty}$ structure $(X, \xi)$. The assertion that $\theta: C X \rightarrow X$ is a map of $\mathcal{G}$-spaces can be written as a commutative distributivity diagram

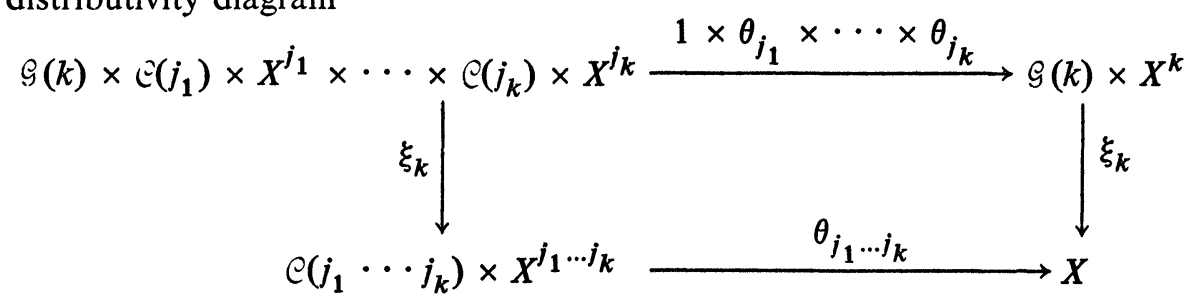

where the map $\xi_{k}$ on the left is as specified in $\$ 9$.

Chases of special cases of the diagram lead to explicit formulas for the computation of \# and the $\tilde{Q}^{r}$ in terms of $*$ and the $Q^{r}$. As an obvious example, with $k=2, j_{1}=2$, and $j_{2}=1$, we get

$$
(x * y) \# z=\sum(-1)^{\operatorname{deg} z^{\prime} \operatorname{deg} y}\left(x \# z^{\prime}\right) *\left(y \# z^{\prime \prime}\right),
$$

where the coproduct on $z$ is given by $\psi z=\Sigma z^{\prime} \otimes z^{\prime \prime}$. Less obviously, with $k=2, j_{1}=p$, and $j_{2}=1$, we get

\footnotetext{
${ }^{3}$ Wellington recently found a gap in Curtis' proof; the quoted result may be false.
} 


$$
\left(Q^{r} x\right) \# y=\sum_{i} Q^{r+i}\left(x \# P_{*}^{i} y\right) .
$$

The formulas for the $\hat{Q}^{r}$ are less tractable. With $k=p$ and $j_{1}=\cdots=j_{p}=$ 2, we get a "mixed Cartan formula" for the evaluation of $\tilde{Q}^{r}(x * y)$. When $p=2$, this reads

$$
\tilde{Q}^{r}(x * y)=\sum_{i+j+k=r} \sum \sum \hat{Q}^{i} x^{\prime} * Q^{j}\left(x^{\prime \prime} \# y^{\prime}\right) * \tilde{Q}^{k} y^{\prime \prime},
$$

the extra summations being over the terms of $\psi x$ and $\psi y$. When $p>2$, the formula is considerably more complicated. Finally, with $k=p$ and $j_{1}$ $=\cdots=j_{p}=p$, we get "mixed Adem relations" for the evaluation of $\tilde{Q}^{r} Q^{s} x$. If $p=2$ and $x=$ [1], the multiplicative identity of $H_{*} X$, these read

$$
\tilde{Q}^{r} Q^{s}[1]=\sum_{j=0}^{s}(r-s-1, s-j) Q^{j}[1] * Q^{r+s-j}[1] \text { for } r>s \geqslant 0
$$

The relations for general $x$ are rather convoluted, and the relations in the case $p>2$ are positively labyrinthine.

When $X$ admits an additive conjugation $\chi$, with $\chi[1]=[-1]$, we have the further relations

$$
\begin{gathered}
x \#[-1]=\chi x ; \tilde{Q}^{r}[-1]=Q^{r}[1] *[-1] \text { if } p=2 \text { and } \\
\tilde{Q}^{r}[-1]=0 \text { if } p>2 \text { and } r>0 .
\end{gathered}
$$

Now consider a $\mathcal{G}$-space $(X, \xi)$ and let $X^{+}$be the $\mathcal{G}_{0}$-space obtained by adjoining a disjoint basepoint 0 to $X$. In terms of $*$ and the $Q^{r}$, we have $H_{*} C\left(X^{+}\right) \cong U H_{*}\left(X^{+}\right)$and $H_{*} Q\left(X^{+}\right) \cong G U H_{*}\left(X^{+}\right)$. The general formulas in principle completely determine the product \# and operations $\hat{Q}^{r}$, in the sense that they provide an algorithm for the expression of any product $x \# y$ and any operation $\tilde{Q}^{r} x$ in terms of the given bases. Indeed, $H_{*} C\left(X^{+}\right)$ and $H_{*} Q\left(X^{+}\right)$are the free allowable " $R$-Hopf bialgebra" and " $R$-Hopf bialgebra with conjugation" generated by $H_{*} X$. In more detail, $H_{*} C\left(X^{+}\right)$is generated under $*$ by elements $Q^{I} x$ for $x \in H_{*} X$. Given $\#$ and the $\tilde{Q}^{r}$ on $H_{*} X$, all products $Q^{I} x y$ for $x, y \in H_{*} X$, can be evaluated by (1) and (2), the Nishida and Adem relations for the $Q^{r}$, and induction on the length of $I$, and then all products $Q^{I} x \# Q^{J} y$ can be computed similarly by induction on the length of $J$. The determination of the $Q^{r}$ is analogous. For any space $Y$, write $[a]$ for a component $a \in \pi_{0} Y$ regarded as an element of $H_{0} Y$. $H_{*} Q\left(X^{+}\right)$has the additional generators $[-a]=[a] \#[-1]$ under $*$, but $\#$ and the $Q^{r}$ are still determined in view of (5).

The most important example is $X=\{1\}$, when $X^{+}=S^{0}$. We have seen that \# and the $\tilde{Q}^{r}$ are completely determined on $\tilde{F}=Q S^{0}$ and thus also on $S F$. Unfortunately, they are determined by some of the most incredibly complicated formulas yet to appear in topology. We are faced with the purely algebraic problem of determining the global structure of $H_{*} S F$ from the given element-wise relations.

For the algebra structure, the problem is quite manageable. It was solved by Milgram [51] when $p=2$ (just from (1) and partial information along the lines of (2), since that formula only came later [44]), and by Tsuchiya [80] and myself [44], independently, when $p>2$. 
For the $R$-algebra structure, the problem is drastically more difficult and, in important respects, still unsolved. The best result is due to Madsen [38], [39], who obtained a minimal set of $R$-algebra generators in the case $p=2$. It is a perversity of nature that while each of the steps of his argument admits a generalization valid for all primes, the resulting statements depend on $p$ in such a way that they fail to yield the desired global information when $p>2$.

Since the algebra structure of $H_{*} S F$ is easily described, more detail may not be out of place. Recall that $H_{*} Q S^{0}$ is generated under $*$ by [ \pm 1$]$ and elements $Q^{I}[1]$ for certain admissible sequences $I$. Write $l(I)$ for the length of I. $Q^{I}[1]$ lies in the $p^{k}$-component of $H_{*} Q S^{0}$ if $l(I)=k$, hence $x_{I}=$ $Q^{\prime}[1] *\left[1-p^{k}\right]$ lies in $H_{*} S F$. If $p>2, H_{*} S F$ is the free commutative algebra generated by the $x_{l}$. If $p=2$, the elements $x_{s}$ have square zero and the exterior algebra $E\left\{x_{s}\right\}$ is the image of $H_{*} S O$ in $H_{*} S F$. Define $x_{(s . s)}=$ $Q^{s} Q^{s}[1] *[-3] ; Q^{s} Q^{s}[1]=Q^{s}[1] * Q^{s}[1]$ but the $x_{(s . s)}$ are indecomposable under \#. Here $H_{*} S F$ is the tensor product of $H_{*} S O$ and the polynomial algebra generated by the $x_{(s, s)}$ and the $x_{I}$ with $l(I) \geqslant 2$.

As noted by Milgram [51], when $p=2$ the coalgebra structure of $H_{*} B S F$ follows directly from the algebra structure of $H_{*} S F$. Its algebra structure (due to Madsen [38]) and the additive as well as the Hopf algebra structure of $H_{*} B S F$ when $p>2$ (due to Tsuchiya [80] and myself [44]) are more delicate, requiring use of the mixed Cartan formula. In all cases, $H_{*} B S F$ has an enormous number of generators $\sigma_{*} x_{1}$. As given, they are specified in terms of the additive operations $Q^{I}[1]$, which have no geometric significance. One wants to describe new generators for $H_{*} S F$ in terms of its own operations $\tilde{Q}^{r}$ since the corresponding generators of $H_{*} B S F$ would then come from the action of $\mathcal{E}$ on $B S F$, and the fibration theoretical interpretation of the maps $\xi_{p}: \mathcal{L}(p) \times_{\Sigma_{p}}(B S F)^{p} \rightarrow B S F$ is well understood. I conjectured (in 1968) that if $I=(J, K)$ with $l(K)=2$ and $\tilde{x}_{I}=\tilde{Q}^{J} x_{K}$, then the description of $H_{*} S F$ as an algebra would remain valid with the $x_{l}$ replaced by $\tilde{x}_{l}$. Madsen [38], [39] proved this when $p=2$ and went further by determining which $x_{L}$ with $l(L)=2$ are decomposable in terms of operations $\tilde{Q}^{J} x_{K}$ with $(J, K)$ inadmissible. When $p>2$, the conjecture is still open. ${ }^{4}$ Even when $p=2$, it would appear to be unmanageably difficult to obtain a defining set of relations for $H_{*} S F$ as an $R$-algebra.

The complexity makes computation of $H_{*}\left(B_{i} S F\right)$ for all $i$ prohibitively difficult, but attempts are being made to compute the cohomology of the spectrum BSF.

Notes. See Madsen [39] when $p=2$ and [20, II, §§1-6] for details and related results. Madsen relied on the mixed Cartan formula, due to him when $p=2$, and on Kochman's calculation [33] of the homology operations in $H_{*} S O$. The mixed Adem relations came later and were obtained in stages by Tsuchiya [82] and myself.

23. The homology of matrix groups; $B C \rightarrow B S F \rightarrow B J_{\otimes}^{\delta}$. Recall that we reduced analysis of $B$ Top at $p>2$ to analysis of $B C$ in $\S 18$ and that we reduced analysis of $B C$, at any prime, to analysis of $e: S F \rightarrow J_{\otimes}^{\delta}$ in $\S 19$. We have explained how $H_{*} S F$ can (in principle) be calculated as an $R$-algebra. The requisite formulas depended ultimately on calculations having to do with the homology of symmetric groups. Among other things, we describe here

\footnotetext{
${ }^{4}$ This contradicts the main theorem of [82]; unfortunately, the proof there contains a gap.
} 
how $H_{*} J_{\otimes}^{\delta}$ can (in principle) be calculated as an $R$-algebra. $J_{\otimes}^{\delta}$ is the 1-component of $\Gamma B \vartheta k_{r}$ (or, equivalently, $\Gamma B \mathcal{G} \mathfrak{L} k_{r}$ ) if $p>2$ and of $\Gamma B \mathfrak{q}\left(k_{3}\right.$ if $p=2$. The additive operations $Q^{r}$ on these group completions are best analyzed by diagram chasing on the level of the homology of groups, and the operations $\tilde{Q}^{r}$ are then computable by the formulas of the previous section. The same diagrams serve to compute the homology operations for such other spaces derived from matrix groups as $B O$ and $B U$, the 0 -components of the spaces $\Gamma B \theta \simeq B O \times Z$ and $\Gamma B \mathcal{U} \simeq B U \times Z$ derived from the classical bipermutative categories of orthogonal and unitary groups.

Let $\mathcal{G}$ be a subbipermutative category of $\mathcal{G} \mathfrak{L} k$ for some (topological) field $k$. Let $\pi$ be cyclic of order $p$ and suppose given a homomorphism $\zeta$ : $\pi \rightarrow G(1) \subset \mathrm{GL}(1, k)$. It is very often the case that much of $H_{*} \Gamma B \mathcal{G}$ is generated under $*$ by $[-1] \in H_{*} \Gamma_{-1} B \mathcal{G}$ and the images $v_{i} \in H_{*} \Gamma_{1} B \mathcal{G}$ of the standard basis elements $e_{i}$ of $H_{i} B \pi$. For example, consider $\mathfrak{C}$ with $p=2$. Here $\pi=O(1)$ and it is classical that $H_{*} B O=P\left\{v_{i} *[-1]\right\}$. Similarly, for any $p$, if $\zeta$ embeds $\pi$ in the circle group $U(1)$, then $H_{*} B U=P\left\{v_{2 i} *[-1]\right\}$.

The additive action $\theta_{p}: \mathscr{D}(p) \times_{\Sigma_{p}} B G(n)^{p} \rightarrow B G(p n)$ of $\S 8$ is the classifying map of the natural inclusion of the wreath product $\Sigma_{p} \int G(n)$ in $G(p n)$. Map $\pi \times \pi$ to $\Sigma_{p} \int G(1)$ via the standard inclusion $\pi \rightarrow \Sigma_{p}$ on the first factor and the composite of $\zeta^{p}$ and the diagonal map $\pi \rightarrow \pi^{p}$ on the second factor. Up to conjugation by a certain matrix $\gamma \in \mathrm{GL}(p, A)$, which in practice lies in the normalizer of $G(p)$, the composite homomorphism $\pi \times \pi \rightarrow \pi \int G(1) \rightarrow$ $G(p)$ breaks up into the sum of $p$ homomorphisms $\pi \times \pi \rightarrow G(1)$, each of which is easy to analyze on homology. There results a formula for calculation of the operations $Q^{r} v_{s}$. When $p=2$, it reads

$$
Q^{r} v_{s}=\sum_{j=0}^{s}(r-s-1, s-j) \gamma_{*}\left(v_{j} * v_{r+s-j}\right) \text { for } r>s \geqslant 0
$$

(and should be compared with (4) of the previous section). If $\gamma \in \mathcal{G}(p)$, then $\gamma_{*}=1$. This holds in the cases $\mathcal{G}=\mathcal{G} \mathfrak{E} k, \mathcal{G}=\mathcal{O}$ with $p=2$, and $\mathcal{G}=\mathscr{Q}$.

Turning to the multiplicative operations, we note that $B G(1)$ is a sub D -space of $B \mathcal{G}$ under $\xi$. Now $\xi: \pi \rightarrow G(1)$, as a map of Abelian groups, is a map of $\Re$-spaces, and the $\mathscr{D}$-action on $G(1)$ is just the pullback of the $\Re$-action. The Pontryagin algebra of $B \pi$ is known and its homology operations are trivial. Thus the products $v_{s} \# v_{t}$ are known and $\tilde{Q}^{r} v_{s}=0$ unless $r=s=0$. By formulas (1) and (5) and the mixed Cartan formula of the previous section, the $*$-subalgebra of $H_{*} B \mathcal{G}$ generated by $[-1]$ and the $v_{i}$ is closed under \# and is closed under the $Q^{r}$ and $\tilde{Q}^{r}$ if $\gamma_{*}=1$; indeed, \# and the $\tilde{Q}^{r}$ are completely determined in terms of $*$ and the $Q^{r}$.

In particular, the procedure just sketched completely determines both $R$-algebra structures on $H_{*} \Gamma B \vartheta$ for $p=2$ and on $H_{*} \Gamma B$ Q for all $p$.

Now consider $J^{\delta}$ and $J_{\otimes}^{\delta}$. First consider $p>2$, where $J^{\delta}=\Gamma_{0} B \mathscr{G} \mathscr{E} k_{r}$. Quillen's calculations [64] determine $H_{*} J^{\delta}$ as a quotient of $H_{*} \Gamma_{0} B \mathscr{S} \mathscr{L} k_{r}(\mu)$, where $k_{r}(\mu)$ is the extension of degree $p-1$ over $k_{r}$ obtained by adjoining all $p$ th roots of unity in the algebraic closure $\bar{k}_{q}\left(r=q^{a}\right.$ is as in $\left.\$ 17\right)$. The procedures discussed above apply directly to

$$
H_{*} \Gamma_{0} B \mathcal{G} \mathfrak{L} k_{r}(\mu)=P\left\{v_{2 i} *[-1]\right\} \otimes E\left\{v_{2 i-1} *[-1]\right\} .
$$

On the other hand, the homological proof of the infinite loop splitting 
$J^{8} \times C \simeq S F$ discussed in $\$ 19$ gives

$$
H_{*} J^{\delta}=P\left\{Q^{s}[1] *[1-p]\right\} \otimes E\left\{\beta Q^{s}[1] *[1-p]\right\}
$$

and determines $\alpha_{*}^{\delta}: H_{*} J^{\delta} \rightarrow H_{*} S F$ explicitly in terms of this description. Comparison of the two sets of generators for $H_{*} J^{\delta}$ allows determination of the operations $Q^{r}\left(\beta^{\varepsilon} Q^{s}[1] *[1-p]\right)$. We have the exponential infinite loop equivalence $e \alpha_{p}^{\delta}: J^{\delta} \rightarrow J_{\otimes}^{\delta}$, and we can therefore read off the operations $\tilde{Q}^{r}$ on $H_{*} J_{\otimes}^{\delta}$. Unfortunately, this information is not very useful because of our incomplete global control on $H_{*} S F$ as an $R$-algebra. However, since $e$ : $S F \rightarrow J_{\otimes}^{\delta}$ is a component of a map of $E_{\infty}$ ring spaces, it preserves the additive as well as the multiplicative operations, and we can obtain precise calculational control on $e_{*}$ from knowledge of the additive operations. On the classifying space level, these methods yield complete information on $H_{*} B C$ and how it sits in $H_{*} B S F$. Via $\$ 18$, this translates to yield a complete analysis of $H_{*} B S$ Top and of the natural map from it to $H_{*} B S F$.

Next consider $p=2$. Here analysis of $J^{\delta}$ requires prior analysis of $\Gamma B \mathcal{O}_{3}$. Its 0-component, denoted $J O^{\delta}$, is equivalent to the fibre of $\psi^{3}-1: B O \rightarrow$ $B S O$, and the Serre spectral sequence of $S O \rightarrow J O^{\delta} \rightarrow B O$ collapses. We have $O\left(1, k_{3}\right)=\pi$, and $H_{*} J O^{\delta}=H_{*} S O \otimes P\left\{v_{i} *[-1]\right\}$ where the elements $v_{i}$ are those considered in our general discussion above. The interesting fact is that the splitting of $H_{*} J O^{\delta}$ is not a splitting of $R$-algebras. The operations $Q^{r}\left(v_{s} *[-1]\right)$ involve elements in $H_{*} S O$, and this phenomenon seems to be undetectable by purely $K$-theoretical study of $\psi^{3}-1$ and JO. In fact, what makes this nonsplitting of $R$-algebras possible is that the element $\gamma \in \mathrm{GL}\left(2, k_{3}\right)$ which enters into formula (1) cannot be chosen in $O\left(2, k_{3}\right)$. Explicit calculation of $\gamma_{*}$ leads to a complete determination of $H_{*} J O^{\delta}$ and $H_{*} J^{\delta}$ as $R$-algebras, and this allows computation of $e_{*}: H_{*} Q_{0} S^{0} \rightarrow H_{*} J^{\delta}$. In the absence of an exponential equivalence $J^{\delta} \rightarrow J_{\otimes}^{\delta}$, it turns out that $e_{*}$ : $H_{*} S F \rightarrow H_{*} J_{\otimes}^{\delta}$ is most efficiently analyzed by translation to 1 -components. An attractive formulation of part of the result is that the minimal set of $R$-algebra generators for $H_{*} S F$ found by Madsen maps onto a minimal set of algebra generators for the complement of $H_{*} \operatorname{Spin}_{\otimes}$ in $H_{*} J_{\otimes}^{\delta} \cong H_{*} \operatorname{Spin}_{\otimes} \otimes$ $\mathrm{H}_{*} B O_{\otimes}$. Again, on the classifying space level, complete information on the homological behavior of the infinite loop fibration $B C \rightarrow B S F \rightarrow B J_{\otimes}^{\delta}$ is obtained. While the detailed results are too complicated to summarize here, one concrete consequence may be worth mentioning. By use of Madsen's work on the higher torsion in $B S F$ [40], the mod 2 calculation allows computation of the maps of Bockstein spectral sequences induced by the fibration above. Madsen had shown that, in cohomology with $Z_{8}$ coefficients, there are classes in $H^{4 i} B S F$ which restrict in $H^{4 i} B S O$ to the mod 8 reductions of the Pontryagin classes. Our results give that there exists such a class in the image of $H^{4 i} B J_{\otimes}^{\delta}$ if and only if $i$ is a power of 2 . This is a surprising, and negative, fact: it had been expected that this image would yield canonical $Z_{8}$ Pontryagin classes in $H^{*} B S F$.

NoTES. See [20, II, \$\$7-13] for complete results. The procedure for computing the $Q^{r} v_{s}$, which is the key to these calculations, is due to Priddy [60], the details when $p>2$ having been worked out by Moore [54]. The first computation of the additive homology operations on $H_{*} B O$ and $H_{*} B U$ was due to Kochman [33], his results being less precise in homology but more precise 
in cohomology than those presented here. In particular, he obtains precise fomulas for the dual operations $Q_{*}^{r}$ on the Stiefel-Whitney and Chern classes (among other results). Herrero [25] working prior to the introduction of $E_{\infty}$ ring spaces, first computed the multiplicative operations for $B O \times Z$ and $B U \times Z$. The calculation of $H_{*} \Gamma B \Theta k_{3}$, with its homology operations, is a very special case of the comprehensive set of calculations of the homology of the classical groups of finite fields obtained by Fiedorowicz and Priddy [24].

The first (and much less precise) calculations of $H_{*} B$ Top when $p>2$ were due to Tsuchiya [81] and myself [44], independently. The calculation of $H_{*} B$ Top when $p=2$ has been carried out by Brumfiel, Madsen, and Milgram [18], the essential point being the surgery theoretical determination of the map $H_{*} F \rightarrow H_{*}(F /$ Top). Madsen's calculation [38] of the homology operations in $H_{*} F /$ Top was the key to determination of the algebra structure of $H_{*} B$ Top and to the calculation of $H_{*} B$ Top $\rightarrow H_{*} B(F /$ Top) in [41].

The analysis of $H_{*} B$ Top for $p>2$ discussed here has been used by Ligaard, Mann, Milgram, and myself to compute the $E_{2}$-term of the Adams spectral sequence converging to $\pi_{*} M S$ Top.

24. The homology of $n$-fold loop spaces. An $n$-fold loop space clearly carries a portion of the internal structure present on an infinite loop space, and this structure leads to a more complicated theory of "unstable" homology operations. Cohen [20, III] has given a complete analysis, and we summarize his results here.

The source of the complexity is already visible on the single loop space level. $H_{*} \Omega X$ is an algebra, generally not commutative, and thus has a $p$ th power operation $\xi_{0}$ and a commutator operation $\lambda_{0}$ under which it is a restricted Lie algebra. Of course, $\xi_{0}$ is not additive, the Cartan formula for $\xi_{0}(x y)$ involves $\lambda_{0}$, and there is a Cartan formula for $\lambda_{0}\left(x x^{\prime}, y y^{\prime}\right)$, a Jacobi identity for iteration of $\lambda_{0}$, and a formula for the evaluation of $\lambda_{0}\left(x, \xi_{0} y\right)$ : Clearly $H_{*} \Omega^{n+1} X$ should admit operations $\xi_{n}$ and $\lambda_{n}$, under which it is some sort of analog of a restricted Lie algebra, such that $\xi_{n}$ suspends to $\xi_{0}$ and $\lambda_{n}$ suspends to $\lambda_{0}$ in $H_{*} \Omega X$. Since $\Omega^{n+1} X$ is also an $(i+1)$-fold loop space for $0 \leqslant i<n$, it should have operations $\xi_{i}$ and $\lambda_{i}$. Just as $\lambda_{0}$ vanishes by commutativity in a 2nd loop space, so all $\lambda_{i}$ for $i<n$ should vanish in an $(n+1)$-fold loop space. But then the $\xi_{i}$ for $i<n$ should be additive and should satisfy the Cartan formula, and thus ought to behave just like the homology operations on infinite loop spaces already discussed. Moreover, there should be commutation formulas among all these operations (Adem relations) and between them and the product and coproduct (Cartan formulas), the Steenrod operations (Nishida relations), and the conjugation.

The appropriate context for this development is that of $\vec{E}_{n+1}$-spaces, where $\mathcal{C}_{n+1}$ is the little cubes operad of $\S 2$. Given such a space $(X, \theta)$, the maps $\theta_{p}$ : $e_{n+1}(p) \times_{\Sigma_{p}} X^{p} \rightarrow X$ give rise to all requisite operations. The commutative diagrams given by the definition of an operad action (compare the diagram in \$20) give all commutation formulas among the operations and between the operations and the product (internal Cartan formulas). The external Cartan formulas are obtainable by easier diagram chases, and they imply the commutation formulas between the operations and the coproduct by naturality; they do not imply the internal Cartan formulas since the product $X \times X \rightarrow X$ 
is only a map of $e_{n}$-spaces, not of $e_{n+1}$-spaces (even up to homotopy). Computation of the Steenrod operations in $H_{*}\left(e_{n+1}(p) \times_{\Sigma_{p}} X^{p}\right)$ yields the Nishida relations by naturality. Commutation of the operations with the conjugation when $X$ is an $(n+1)$-fold loop space is proven by use of an appropriate involution of the little cubes operad.

The essential first step of the program is calculation of

$$
H_{*}\left(\bigodot_{n+1}(p) \times_{\Sigma_{p}} X^{p}\right) .
$$

The space $\bigodot_{n+1}(p)$ may be replaced by the configuration space $F\left(R^{n+1}, p\right)$, and the key point is the determination of the homology of the braid space $B\left(R^{n+1}, p\right)=F\left(R^{n+1}, p\right) / \Sigma_{p}$. When $n=1$, this space is a $K\left(B_{p}, 1\right)$, where $B_{p}$ is Artin's group of braids on $p$ strings [8], hence the terminology. Work of Fadell and Neuwirth [23] gives the starting point for the calculation of $H^{*} F\left(R^{n+1}, p\right)$ as a $\Sigma_{p}$-algebra, and a careful analysis of the spectral sequence of the covering $F\left(R^{n+1}, p\right) \rightarrow B\left(R^{n+1}, p\right)$ leads to the required calculation of $H_{*} B\left(R^{n+1}, p\right)$. Of course, when $n=1$, this gives the homology of the classical braid groups. Proofs of commutation formulas require information on $H_{*}\left(\bigodot_{n+1}(k) \times_{\Sigma_{k}} X^{k}\right)$ for certain values of $k$ other than $p$ and on certain of the homomorphisms $\gamma_{*}$ determined by the structural maps of $\bigcup_{n+1}$.

Actually, the details are much more devious than the straightforward outline above would indicate. Determination of many of the commutation formulas along the lines indicated would be prohibitively difficult, and we have not yet mentioned the most crucial property of the operations, namely commutation with the suspension $\sigma_{*}: \tilde{H}_{*} \Omega^{n+1} X \rightarrow H_{*} \Omega^{n} X$.

The rigorous development interweaves the program above with calculation of $H_{*} \Omega^{n+1} \Sigma^{n+1} X$ along the same lines as the calculation of $H_{*} Q X$ sketched in $\$ 21$, comparison of these two calculations, and exploitation of the geometric diagrams constructed in [45] for the inductive proof of the approximation theorem of $\S 4$.

$H_{*} \Omega^{n+1} \Sigma^{n+1} X$, with all structure in sight, turns out to be the appropriate free functor (with conjugation) on $H_{*} X$. By an analog of the calculation of $H_{*} C X$ sketched in $\$ 21$, and use of the equivalence $C_{n+1} X \rightarrow \Omega^{n+1} \Sigma^{n+1} X$ for connected $X$, Cohen also proved that, for any $X, H_{*} C_{n+1} X$ is the appropriate free functor (without conjugation) on $H_{*} X$ and so completed the proof of the approximation theorem of $\S 4$.

One other calculation of Cohen [20, IV] should be mentioned. When $p=2$ or when $p>2$ and $n$ is odd, the natural map from $H_{*} S F(n)$ to $H_{*} S F$ is a monomorphism because in these cases the operation $\lambda_{n-1}$ on $H_{*} \Omega^{n} S^{n}$ is trivial. When $n$ is even and $p>2$, there is a large kernel. Cohen proved that $H_{*} S F(n)$ is free commutative on specified generators. The essential point is commutativity. Since $S F(n)$ is not homotopy commutative, the proof required explicit calculation of commutators and in fact required use of every one of the commutation formulas between operations. In contrast to the stable case, determination of $H_{*} B S F(n)$ does not follow directly, due to lack of internal structure on $B S F(n)$, and is work in progress.

Notes. When $p=2$, Araki and Kudo [7] constructed all requisite one-variable operations on $H_{*} \Omega^{n} X$. The $\lambda_{n}$ were introduced by Browder [16]. Dyer and Lashof [22] introduced some of the one-variable operations when $p>2$, by use of skeleta of $B \Sigma_{p}$, but it is impossible to so construct all of the 
operations. Milgram's calculation [50] of $H_{*} \Omega^{n} \Sigma^{n} X$ as an algebra implicitly gave all operations, but not in a form usable for such detailed calculations as those discussed here.

Conclusion. The theory and applications that we have discussed are reasonably complete, although there are numerous loose ends. On the theoretical side, for example, precise comparisons between the black box discussed here and those developed by other authors would be of interest.

In the area of calculations of characteristic classes, and more so in the concomitant area of calculations of cobordism classes (of topological bundles and Poincare duality spaces), there are a number of basic computations still to be made. There also remain unsolved problems in the area of descriptive analysis on the infinite loop space level of the classifying spaces of geometric topology (notably concerning $B$ Top at 2 and the Adams conjecture away from 2).

Applications of the full strength of infinite loop space theory to algebraic $K$-theory seem likely to lie far in the future. The theory's calculational power lies in applications to known homotopy types and their deloopings, and it is precisely the determination of such basic underlying homotopy types as that of $(B \mathcal{G} \mathscr{L Z})^{+}$which is most needed (and seems most intractable) in this area.

One direction in which very much more work needs to be done is the exploration on the calculational level of the connections between infinite loop space theory and stable homotopy theory. From the theoretical point of view, it is well understood that these are two sides of the same coin: with the appropriate morphisms, the categories of (grouplike) $E_{\infty}$ spaces and of connective spectra are equivalent. However, from the calculational point of view, there is a marked disjunction between the techniques used for analysis of the two sides of the coin. For example, there is no known calculational route from the homology of a spectrum to the homology of its zeroth space, and the known theoretical calculational route the other way is at best tortuous.

As we have pointed out in several places, substantial homotopy theoretical results have already been obtained by infinite loop space techniques. It is my hope that these are just the beginning, and that much new information will come when we learn how the rich space level structures described here can effectively be exploited for calculations in stable homotopy theory.

\section{BIBLIOGRAPHY}

1. J. F. Adams, On the groups $J(X)$. I-IV, Topology 2 (1963), 181-195; ibid. 3(1965), 137-171; ibid. 3 (1965), 193-222; ibid. 5 (1966), 21-71. MR 28 \#2553; 33 \#6626, 6627, 6628.

2. L Lectures on generalized cohomology, Category Theory, Homology Theory and Their Applications, III (Battelle Inst. Conf., Seattle, Wash., 1968, Vol. Three), Lecture Notes in Math., vol. 99, Springer, Berlin, 1969, pp. 1-138. MR 40 \#4943.

3. J. F. Adams and S. B. Priddy, Uniqueness of BSO, Proc. Cambridge Philos. Soc. (to appear).

4. D. W. Anderson, Simplicial K-theory and generalized homology theories (preprint).

5. - There are no phantom cohomology operations in $K$-theory (preprint).

6. D. W. Anderson and L. Hodgkin, The K-theory of Eilenberg-Mac Lane complexes, Topology 7 (1968), 317-329. MR 37 \#6924.

7. S. Araki and T. Kudo, Topology of $H_{n}$-spaces and $H$-squaring operations, Mem. Fac. Sci. Kyūsyū Univ. Ser. A 10 (1956), 85-120. MR 19, 442. 
8. E. Artin, Theory of braids, Ann. of Math. (2) 48 (1947), 101-126. MR 8, 367.

9. M. F. Atiyah, R. Bott and A. Shapiro, Clifford modules, Topology 3 (1964), suppl. 1, 3-38. MR 29 \#5250.

10. M. G. Barratt, A free group functor for stable homotopy, Proc. Sympos. Pure Math., Vol. 22, Amer. Math. Soc., Providence, R. I., 1971, pp. 31-35. MR 48 \#3043.

11. M. G. Barratt and P. J. Eccles, $\Gamma^{+}$-structures. I-III, Topology 13 (1974), 25-45; ibid. 13 (1974), 113-126; ibid. 13 (1974), 199-207. MR 50 \# 1234a, b, c.

12. J. Beck, On $H$-spaces and infinite loop spaces, Category Theory, Homology Theory and Their Applications, III (Battelle Inst. Conf., Seattle, Wash., 1968, Vol. Three), Lecture Notes in Math., vol. 99, Springer, Berlin, 1969, pp. 139-153. MR 40 \#2079.

13. J. M. Boardman and R. M. Vogt, Homotopy-everything H-spaces, Bull. Amer. Math. Soc. 74 (1968), 1117-1122. MR 38 \#5215.

14. Homotopy invariant algebraic structures on topological spaces, Lecture Notes in Math., vol. 347, Springer, New York, 1973.

15. A. K. Bousfield and D. M. Kan, Homotopy limits, completions, and localizations, Lecture Notes in Math., vol. 304, Springer-Verlag, Berlin and New York, 1972. MR 51 \# 1825.

16. W. Browder, Homology operations and loop spaces, Illinois J. Math. 4 (1960), 347-357. MR $22 \# 11395$.

17. , Surgery on simply-connected manifolds, Ergebnisse math. Grenzgebiete, Bd. 65 , Springer-Verlag, New York, 1972. MR 50 \# 11272.

18. G. Brumfiel, I. Madsen and R. J. Milgram, PL characteristic classes and cobordism, Ann. of Math. (2) 97 (1973), 82-159. MR 46 \#9979.

19. F. Cohen, Splitting certain suspensions via self-maps. Illinois J. Math. (to appear).

20. F. Cohen, T. Lada and J. P. May, The homology of iterated loop spaces, Lecture Notes in Math. vol. 533, Springer-Verlag, Berlin and New York, 1976.

21. E. B. Curtis, The Dyer-Lashof algebra and the $\Lambda$-algebra, Illinois J. Math. 19 (1975), 231-246.

22. E. Dyer and R. K. Lashof, Homology of iterated loop spaces, Amer. J. Math. 84 (1962), 35-88. MR 25 \# 4523.

23. E. Fadell and L. Neuwirth, Configuration spaces, Math. Scand. 10 (1962), 111-118. MR 25 \#4537.

24. Z. Fiedorowicz and S. Priddy, Loop spaces and finite orthogonal groups, Bull. Amer. Math. Soc. 81 (1975), 700-702. MR 51 \#11487.

25. M. Herrero, Homology operations on $H_{*}(B U \times Z)$ and $H_{*}(B O \times Z)$ related to the tensor product of vector bundles, Thesis, Univ. of Chicago, 1972.

26. L. Hodgkin, The $K$-theory of some well-known spaces. I: $Q S^{0}$, Topology 11 (1972), 371-375. MR 48 \#9701.

27. L. Hodgkin and V. P. Snaith, The K-theory of some more well-known spaces (preprint).

28. J. R. Isbell, On coherent algebras and strict algebras, J. Algebra 13 (1969), 299-307. MR 40 \#2729.

29. L. Jones, Patch spaces: a geometric representation for Poincaré spaces, Ann. of Math. (2) 97 (1973), 276-306. MR 47 \#4269.

30. D. S. Kahn, Cup-i products and the Adams spectral sequence, Topology 9 (1970), 1-9. MR 40 \#6552.

31. Homology of the Barratt-Eccles decomposition maps, Notas de Matematicas y Simposià, No 1., Soc. Mat. Mexicana, 1975.

32. D. S. Kahn and S. B. Priddy, Applications of the transfer to stable homotopy theory, Bull. Amer. Math. Soc. 78 (1972), 981-987. MR 46 \#8220.

33. S. O. Kochman, Homology of the classical groups over the Dyer-Lashof algebra, Trans. Amer. Math. Soc. 185 (1973), 83-136. MR 48 \#9719.

34. M. Laplaza, Coherence for distributivity, Coherence in Categories, Lecture Notes in Math., vol. 281, Springer, Berlin, 1972, pp. 29-65. MR 49 \#379a.

35. N. Levitt and J. W. Morgan, Transversality structures and P.L. structures on spherical fibrations, Bull. Amer. Math. Soc. 78 (1972), 1064-1068. MR 47 \#2602.

36. H.J. Ligaard, Infinite loop maps from $\mathrm{SF}$ to $\mathrm{BO}_{\otimes}$ at the prime 2, Illinois J. Math. (to appear)

37. S. Mac Lane, Categorical algebra, Bull. Amer. Math. Soc. 71 (1965), 40-106. MR 30 \#2053. 
38. I. Madsen, On the action of the Dyer-Lashof algebra in $H_{*}(G)$ and $H_{*}(G /$ Top), Thesis, Univ. of Chicago, 1970.

39. , On the action of the Dyer-Lashof algebra in $H_{*}(G)$, Pacific J. Math. 60 (1975), 235-275.

40. - Higher torsion in SG and BSG, Math. Z. 143 (1975), 55-80. MR 51 \#11503.

41. I. Madsen and R. J. Milgram, The universal smooth surgery class, Comment. Math. Helv. 50 (1975), 281-310.

42. I. Madsen, V. P. Snaith and J. Tornehave, Infinite loop maps in geometric topology, Math. Proc. Cambridge Philos. Soc. (to appear).

43. J. P. May, A general algebraic approach to Steenrod operations, The Steenrod Algebra and Its Applications (Proc. Conf., Battelle Memorial Inst., Columbus, Ohio, 1970), Lecture Notes in Math., vol. 168, Springer, Berlin, 1970, pp. 153-231. MR 43 \#6915.

44. - Homology operations on infinite loop spaces, Proc. Sympos. Pure. Math., Vol. 22. Amer. Math. Soc. Providence, R. I., 1971. pp. 171-185. MR 47 \# 7740.

45. _ The geometry of iterated loop spaces, Lecture Notes in Math. Vol. 271, Springer,

New York, 1972.

46. —,$E_{\infty}$ spaces, group completions, and permutative categories, New Developments in Topology (Proc. Sympos. Algebraic Topology, Oxford, 1972), London Math. Soc. Lecture Note Ser., no. 11, Cambridge Univ. Press, London, 1974, pp. 61-93. MR 49 \#3915.

47. Classifying spaces and fibrations, Mem. Amer. Math. Soc. 1 (1975), issue 1, no. 155. MR 51 \#6806.

48. (with contributions by F. Quinn, N. Ray and J. Tornehave), $E_{\infty}$ ring spaces and $E_{\infty}$ ring spectra, Lecture Notes in Math.,vol 577, Springer-Verlag, Berlin and New York, 1977.

49. - The homotopical foundations of algebraic topology, Monograph London Math.

Soc., Academic Press, London (in preparation).

50. R. J. Milgram, Iterated loop spaces, Ann. of Math. (2) 84 (1966), 386-403. MR 34 \#6767.

51. - The mod 2 spherical characteristic classes, Ann. of Math. (2) 92 (1970), 238-261.

MR 41 \#7705.

52. _ Group representations and the Adams spectral sequence, Pacific J. Math. 41 (1972),

157-182. MR 46 \#3598.

53. , Unstable homotopy from the stable point of view, Lecture Notes in Math., vol. 368, Springer-Verlag, Berlin and New York, 1974. MR 50 \# 1235.

54. D. Moore, On homology operations for the classifying spaces of certain groups, Thesis, Northwestern Univ., Evanston, Ill., 1974.

55. M. Nakaoka, Decomposition theorem for homology groups of symmetric groups, Ann. of Math. (2) 71 (1960), 16-42. MR 22 \#2989.

56. - Homology of the infinite symmetric group, Ann. of Math. (2) 73 (1961), 229-257. MR 24 \# A1721.

57. G. Nishida, Cohomology operations in iterated loop spaces, Proc. Japan Acad. 44 (1968), 104-109. MR 39 \#2156.

58. Japan 25 (1973), 707-732. MR 49 \#6236.

59. F. P. Peterson, The Mod $p$ homotopy type of BSO and F/PL, Bol. Soc. Mat. Mexicana (2) 14 (1969), 22-27. MR 41 \#540.

60. S. B. Priddy, Dyer-Lashof operations for the classifying spaces of certain matrix groups, Quart. J. Math. Oxford Ser. (2) 26 (1975), no. 102, 179-193. MR 51 \#11505.

61. D. Quillen, The Adams conjecture, Topology 10 (1970), 67-80. MR 43 \#5525.

62. , On the cohomology and $K$-theory of the general linear groups over a finite field, Ann. of Math. (2) 96 (1972), 552-586. MR 47 \#3565.

63. Higher K-theory for categories with exact sequences, New Developments in Topology (Proc. Sympos. Algebraic Topology, Oxford, 1972), London Math. Soc. Lecture Note Ser., no. 11, Cambridge Univ. Press, London, 1974, pp. 95-103. MR 49 \#384.

64. _ Higher algebraic $K$-theory. I, Algebraic $K$-Theory. I: Higher $K$-Theories (Proc. Conf. Seattle Res. Center, Battelle Memorial Inst., 1972), Lecture Notes in Math., vol. 341, Springer, Berlin, 1973, pp. 85-147. MR 49 \#2895.

65. F. Quinn, Surgery on Poincare and normal spaces, Bull. Amer. Math. Soc. 78 (1972), 262-267. MR 45 \#6014.

66. G. Segal, Configuration-spaces and iterated loop-spaces, Invent. Math. 21 (1973), 213-221. MR 48 \#9710. 
67. G. Segal, Categories and cohomology theories, Topology 13 (1974), 293-312. MR 50 \# 5782.

68. J.-P. Serre, Cohomologie modulo 2 des complexes d'Eilenberg-Mac Lane, Comment. Math.

Helv. 27 (1953), 198-232. MR 15, 643.

69. V. P. Snaith, A stable decomposition of $\Omega^{n} S^{n} X$, J. London Math. Soc. (2) 7 (1974), 577-583, MR 49 \#3918.

70. , Dyer-Lashof operations in K-theory, Lecture Notes in Math., vol. 496, Springer, New York, 1975, pp. 103-294.

71. J. D. Stasheff, Homotopy associativity of $H$-spaces. I, II, Trans. Amer. Math. Soc. 108 (1963), 275-312. MR 28 \#1623.

72. N. E. Steenrod (Notes, rev. by D.B.A. Epstein), Cohomology operations, Ann. of Math. Studies, no. 50, Princeton Univ. Press, Princeton, N. J., 1962. MR 26 \#3056.

73. D. Sullivan, Geometric topology seminar, Princeton University (mimeographed notes).

74. , Geometric topology. I: Localization, periodicity, and Galois symmetry, M.I.T. (mimeographed notes).

75. , Genetics of homotopy theory and the Adams conjecture, Ann. of Math. (2) 100 (1974), 1-79.

76. E. Thomas, The generalized Pontrjagin cohomology operations and rings with divided powers, Mem. Amer. Math. Soc. No. 27 (1957), 82 pp. MR 20 \#5474.

77. J. Tornehave, Delooping the Quillen map, Thesis, M.I.T., 1971.

78. _ On BSG and the symmetric groups (preprint).

79. - On the splitting of spherical fibration theory at odd primes (preprint).

80. A. Tsuchiya, Characteristic classes for spherical fiber spaces, Nagoya Math. J. 43 (1971), 1-39. MR 45 \#7736.

81. Characteristic classes for PL micro bundles, Nagoya Math. J. 43 (1971), 169-198. MR 47 \#2614.

82. Homology operations on ring spectrum of $H^{\infty}$ type and their applications, J. Math. Soc. Japan 25 (1973), 277-316. MR 47 \# 4255.

Department of Mathematics, University of Chicago, Chicago, Illinois 60637 